\title{
The misrepresentation of spatial uncertainty in visual search: Single- versus joint-distribution probability cues
}

\author{
Bradley S. Gibson ${ }^{1} \cdot$ Joseph R. Pauszek ${ }^{1} \cdot$ Jamie M. Trost $^{1} \cdot$ Michael J. Wenger ${ }^{2}$ \\ Accepted: 8 September 2020 / Published online: 6 October 2020 \\ (C) The Psychonomic Society, Inc. 2020
}

\begin{abstract}
The present study used information theory to quantify the extent to which different spatial cues conveyed the entropy associated with the identity and location of a visual search target. Single-distribution cues reflected the probability that the target would appear at one fixed location whereas joint-distribution cues reflected the probability that the target would appear at the location where another cue (arrow) pointed. The present study used a novel demand-selection paradigm to examine the extent to which individuals explicitly preferred one type of probability cue over the other. Although both cues conveyed equal entropy, the main results suggested representation of greater target entropy for joint- than for single-distribution cues based on a comparison between predicted and observed probability cue choices across four experiments. The present findings emphasize the importance of understanding how individuals represent basic information-theoretic quantities that underlie more complex decision-theoretic processes such as Bayesian and active inference.
\end{abstract}

Keywords Visual search $\cdot$ Visual perception $\cdot$ Attention: space-based

\section{Introduction}

The synthetic approach to psychological science recommends that theories should be founded on broad, continuous constructs derived from general principles (Cisek, 2019; Hommel et al., 2019). The "free energy principle" in computational neuroscience is one candidate principle that may unify theories of perception, cognition, and action; this principle contends a biological imperative to minimize the uncertainty of an individual's sensory, motoric, and metabolic states based on an internal model capable of predicting those future states (Bogacz, 2017; Clark, 2016; Friston, 2009, 2013; Gershman, 2019; Hutchinson \& Barrett, 2019). The free energy principle therefore predicts that individuals should generally seek to minimize uncertainty in their interactions with the world. Consistent with this general principle, there is strong behavioral and physiological evidence that the brain represents probability distributions and performs inferences based on

Bradley S. Gibson

bgibson@nd.edu

1 Department of Psychology, University of Notre Dame, 390 Corbett Family Hall, Notre Dame, IN, USA

2 Department of Psychology, University of Oklahoma, Norman, OK, USA those distributions (see Ma, 2012, and Pouget, Beck, Ma, \& Latham, 2013, for reviews). However, there is also evidence to suggest that individual behavior can reflect suboptimal inferences arising from incorrect probability distributions as opposed to noise (Beck, Ma, Pitkow, Latham, \& Pouget, 2012).

The present study was designed to examine this biological imperative within the context of visual search, and the extent to which an individual's ability to perform optimal (or ideal) inferences might depend on the structure of underlying probability distributions. Researchers have long known that natural scenes and visual displays can vary in the amount of uncertainty they convey (Eriksen, 1952, 1953, 1955). For instance, the spatial locations of desired objects and events in the outside world are often not perfectly predictable, but they are typically not randomly distributed in the environment either, and individuals appear to utilize knowledge of these imperfect statistical regularities to guide visual search (Shaw \& Shaw, 1977). In the present article, we refer to these imperfect yet stable statistical regularities as expected forms of uncertainty (Bland \& Schaefer, 2012; Yu \& Dayan, 2005).

Within the context of visual search, expected forms of spatial uncertainty have been studied using a variety of different probability cues (Colgate, Hoffman, \& Eriksen, 1973; Eriksen \& Hoffman, 1972; Gibson \& Kingstone, 2006; Jonides, 1981; Posner, 1980). Here we will focus on two types of cues which, for reasons that will become clearer below, we will term 
"single-distribution" and "joint-distribution" probability cues. Single-distribution probability cues typically involve search contexts in which the target appears at one fixed location more often than it appears at other fixed locations. For instance, Jiang, Swallow, Rosenbaum, and Herzig (2013b) divided their search display into four quadrants; the target appeared in the high (or rich) probability quadrant on $50 \%$ of the trials, whereas it appeared in each of the other low (or sparse) probability quadrants on $17 \%$ of the trials. In contrast, jointdistribution probability cues typically involve search contexts in which the target appears at one cued location, which can shift from one trial to the next, more often that it appears at the other uncued locations. For instance, in the spatial cueing paradigm (Posner, 1980; see also Chica, Martín-Arévalo, Botta, \& Lupiáñez, 2014, for a review), a centrally presented spatial cue such as an arrow is typically displayed for several hundred milliseconds before the appearance of a peripheral visual search display, and the target either appears at the cued location or one of the uncued locations. Regardless of the type of cue, the uncertainty of a given task context is represented either by the validity of the location - i.e., the extent to which the target appears at the one fixed location - or the validity of the cue - i.e., the extent to which it accurately points at the target.

Although previous research has examined potential differences in how single-distribution and joint-distribution forms of probability cues might control attention (Geng \& Behrmann, 2002, 2005; Jiang, 2018; Jiang, Swallow, \& Rosenbaum, 2013a), none has examined the more fundamental question concerning the extent to which these different forms of probability cues might impact the representation of uncertainty. Inspired by the work of Eriksen and Hake (1955a, 1955b, Hake \& Eriksen, 1955), the present study used information theory to quantify the expected uncertainty typically encountered in these two paradigms, and it inferred differences in the representation of uncertainty by examining the extent to which individuals explicitly preferred one form of expected uncertainty over the other.

For instance, consider a visual search task in which one of two possible target letters (E vs. U) appeared at one of four possible fixed locations (above vs. below vs. left vs. right). For the sake of convenience, target identity and target location were combined into a single random variable, $T$, which had eight possible values in the present context: $t_{1}=\mathrm{E}$ above, $t_{2}=$ $\mathrm{E}$ below, $t_{3}=\mathrm{E}$ left,$t_{4}=\mathrm{E}$ right,$t_{5}=\mathrm{U}$ above, $t_{6}=\mathrm{U}$ below, $t_{7}$ $=\mathrm{U}$ left, and $t_{8}=\mathrm{U}$ right . Table 1 lists the probability values associated with three single-distribution probability cues in which the target appeared at the above location either $100 \%$, $70 \%$, or $25 \%$ of the time (identical values could also be created for the below, left, and right locations). Of critical interest was the quantity known as Shannon information which represents the average uncertainty (or surprise) in a probability distribution (Cover \& Thomas, 2006; see Stone, 2015, for a tutorial review). The average surprise of the single probability distribution $p(T)$ is called the entropy of $p(T)$ and is represented as $H(T)$. The average entropy is defined as:

$H(T)=\sum_{i=1}^{m_{t}} p\left(t_{i}\right) \log _{2} \frac{1}{p\left(t_{i}\right)}$,

where $m_{t}$ represents the number of different values of T. Note that all logarithms used a base of two in this article, and the corresponding quantities reflect the amount of uncertainty measured in bits, where one bit is the amount of information required to choose between two equally probable alternatives. For instance, there is one bit of target entropy associated with the $100 \%$ valid single-distribution probability cue because the identity of the target remains unknown which is equally likely to be $\mathrm{E}$ or $\mathrm{U}$. The left panel of Fig. 1 shows how the value of target entropy varied across a wide range of location validities; target entropy gradually increased from a minimum of one bit to a maximum of three bits as the single-distribution probability cue decreased from $100 \%$ to $25 \%$ (chance). In other words, the uncertainty of the target's identity and location increased as the single-distribution probability cue became less accurate.

Likewise, a similar target entropy function can be created using joint-distribution probability cues, though the computation of this entropy function is complicated by the fact that the entropy of the target's identity and location in this situation depends on the joint probability between it and a second random variable - cue direction $(C)$ - which had four values in the present context: $c_{1}=$ above, $c_{2}=$ below, $c_{3}=$ left , and $c_{4}=$ right. Table 2 lists the corresponding three joint-distribution probability cues in which the target appeared at the cued location either $100 \%, 70 \%$, or $25 \%$ of the time; the marginal and joint probability distributions are listed in Table 2 as a function of these three joint-distribution probability cues. The average entropies of the two marginal distributions are defined by Eq. 1 ; the average surprise of the joint probability distribution is defined as:

$H(T, C)=\sum_{i=1}^{m_{t}} \sum_{j=1}^{m_{c}} p\left(t_{i}, c_{j}\right) \log _{2} \frac{1}{p\left(t_{i}, c_{j}\right)}$,

where $m_{t}$ and $m_{c}$ represent the number of different values of $T$ and $C$, respectively.

In order to derive the entropy of the target's identity and location in the (joint-distribution) spatial cueing paradigm, the average entropy of the joint probability distribution must be partitioned into three information-theoretic quantities, which are shown in the right panel of Fig. 1 across a wide range of cue validities. Consider first the black region of each bar, which represents the average "mutual information" that is shared between target identity and location and cue direction. Mutual information reflects the average reduction in uncertainty about the target's identity and location that is provided by cue direction, and vice versa. Average mutual information 
Table 1 Simple target entropy associated with three single-distribution probability cues in which the target appeared at the above location $100 \%, 70 \%$, or $25 \%$ of the time. Note that identical values could also be created for the below, left, and right locations

\begin{tabular}{lccc}
\hline & $\begin{array}{l}\text { Location validity }=100 \% \\
\text { Target entropy }=1.00\end{array}$ & $\begin{array}{c}\text { Location validity }=70 \% \\
\text { Target entropy }=2.36\end{array}$ & $\begin{array}{l}\text { Location validity }=25 \% \\
\text { Target entropy }=3.00\end{array}$ \\
\hline E above & .500 & .350 & .125 \\
E right & & .050 & .125 \\
E below & & .050 & .125 \\
E left & .500 & .050 & .125 \\
U above & & .350 & .125 \\
U right & & .050 & .125 \\
U below & & .050 & .125 \\
U left & & .050 & .125 \\
\hline
\end{tabular}

is defined as:

$I(T, \mathrm{C})=H(T)+H(C)-H(T, C)$,

where $H(T)$ and $H(C)$ represent the average marginal entropies associated with the variables $T$ and $C$, respectively. Note that average mutual information is equal to zero when $T$ and $C$ are independent, and related only by chance, because $H(T)+H(C)$ $=H(T, C)$ in this case. As can be seen in the right panel of Fig. 1 , mutual information decreased as cue validity decreased from a maximum of $100 \%$ to a minimum of $25 \%$ (chance).

In addition to mutual information, consider also the crosshatched and gray regions of each bar, which represented the two sources of "conditional entropy." Conditional entropy refers to the residual expected uncertainty that remained in each of the random variables after the information provided by the other variable had been accounted for. The crosshatched region of each bar reflected the average uncertainty in target identity and location that remained after knowing cue direction, $H(T \mid C)$ - referred to here as "conditional target entropy"; and, the gray region of each bar reflected the average uncertainty in cue direction that remained after knowing target identity and location, $H(C \mid T)$ - referred to here as "conditional cue entropy." These two forms of average conditional entropy are defined formally as:

$H(T \mid C)=H(T)-I(T, C)$,

and

$H(C \mid T)=H(C)-I(T, C)$.

Note that the existence of conditional cue entropy has typically been overlooked in this paradigm.

Figure 1 shows that the magnitude of simple target entropy (derived from single-distribution probability cues) was equivalent to the magnitude of conditional target entropy (derived from joint-distribution probability cues) across the full range of different probability cues. However, despite the equality of these two forms of target entropy, the simple form can be estimated directly from the target identity and location probability distribution whereas the conditional form can only be

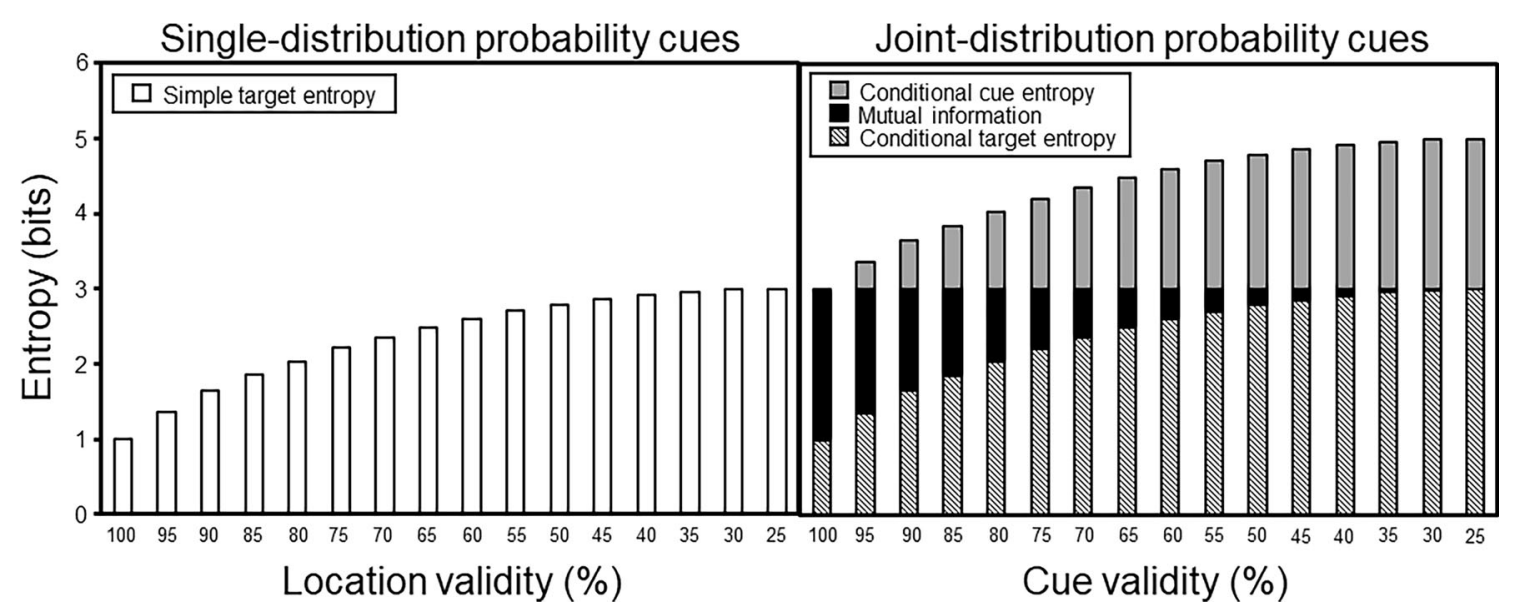

Fig. 1 Average entropy of the single (left panel) and joint (right panel) probability distributions shown as a function of location or cue validity, respectively 
Table 2 Mutual information, conditional cue entropy, and conditional target entropy associated with three joint-distribution probability cues in which the target appeared at the cued location either $100 \%, 70 \%$, or $25 \%$ of the time

\begin{tabular}{|c|c|c|c|c|c|c|c|c|c|c|c|c|c|c|c|}
\hline & \multicolumn{5}{|c|}{$\begin{array}{l}\text { Cue validity }=100 \% \\
\text { Marginal cue entropy }=2.00 \\
\text { Marginal target entropy }=3.00 \\
\text { Joint entropy }=3.00 \\
\text { Mutual information }=2.00 \\
\text { Conditional cue entropy }=0.00 \\
\text { Conditional target entropy }=1.00\end{array}$} & \multicolumn{5}{|c|}{$\begin{array}{l}\text { Cue validity }=70 \% \\
\text { Marginal cue entropy }=2.00 \\
\text { Marginal target entropy }=3.00 \\
\text { Joint entropy }=4.36 \\
\text { Mutual information }=0.64 \\
\text { Conditional cue entropy }=1.36 \\
\text { Conditional target entropy }=2.36\end{array}$} & \multicolumn{5}{|c|}{$\begin{array}{l}\text { Cue validity }=25 \% \\
\text { Marginal cue entropy }=2.00 \\
\text { Marginal target entropy }=3.00 \\
\text { Joint entropy }=5.00 \\
\text { Mutual information }=0.00 \\
\text { Conditional cue entropy }=2.00 \\
\text { Conditional target entropy }=3.00\end{array}$} \\
\hline & $\begin{array}{l}\text { A } \\
\text { cue }\end{array}$ & $\begin{array}{l}\mathrm{R} \\
\text { cue }\end{array}$ & $\begin{array}{l}\text { B } \\
\text { cue }\end{array}$ & $\begin{array}{l}\mathrm{L} \\
\text { cue }\end{array}$ & Marg & $\begin{array}{l}\text { A } \\
\text { cue }\end{array}$ & $\begin{array}{l}\mathrm{R} \\
\text { cue }\end{array}$ & $\begin{array}{l}\text { B } \\
\text { cue }\end{array}$ & $\begin{array}{l}\mathrm{L} \\
\text { cue }\end{array}$ & Marg & $\begin{array}{l}\text { A } \\
\text { cue }\end{array}$ & $\begin{array}{l}\mathrm{R} \\
\text { cue }\end{array}$ & $\begin{array}{l}\text { B } \\
\text { cue }\end{array}$ & $\begin{array}{l}\mathrm{L} \\
\text { cue }\end{array}$ & Marg \\
\hline E above & .125 & & & & .125 & .0875 & .0125 & .0125 & .0125 & .125 & .03125 & .03125 & .03125 & .03125 & .125 \\
\hline E right & & .125 & & & .125 & .0125 & .0875 & .0125 & .0125 & .125 & .03125 & .03125 & .03125 & .03125 & .125 \\
\hline E below & & & .125 & & .125 & .0125 & .0125 & .0875 & .0125 & .125 & .03125 & .03125 & .03125 & .03125 & .125 \\
\hline E left & & & & .125 & .125 & .0125 & .0125 & .0125 & .0875 & .125 & .03125 & .03125 & .03125 & .03125 & .125 \\
\hline U above & .125 & & & & .125 & .0875 & .0125 & .0125 & .0125 & .125 & .03125 & .03125 & .03125 & .03125 & .125 \\
\hline U right & & .125 & & & .125 & .0125 & .0875 & .0125 & .0125 & .125 & .03125 & .03125 & .03125 & .03125 & .125 \\
\hline U below & & & .125 & & .125 & .0125 & .0125 & .0875 & .0125 & .125 & .03125 & .03125 & .03125 & .03125 & .125 \\
\hline U left & & & & .125 & .125 & .0125 & .0125 & .0125 & .0875 & .125 & .03125 & .03125 & .03125 & .03125 & .125 \\
\hline Marg & .25 & .25 & .25 & .25 & 1.00 & .25 & .25 & .25 & .25 & 1.00 & .25 & .25 & .25 & .25 & 1.00 \\
\hline
\end{tabular}

Note $:$ Marg = marginal

$A=$ Above; $R=$ Right $B=$ Below; $L=$ Left

estimated indirectly after it has been partitioned from mutual information and conditional cue entropy in the joint probability distribution.

Given these two distinctly different methods of estimation, the question arises as to whether individuals might prefer the single-distribution method of estimation over the jointdistribution method in the context of visual search. In general, single-distribution probability cues might be preferred because the uncertainty they convey is represented more accurately than joint-distribution probability cues. Two potential differences in the representation of uncertainty are of particular interest. First, the representation of conditional target entropy might change relative to the representation of simple target entropy as a function of cue validity. For instance, the representation of conditional target entropy might be larger than the representation of simple target entropy only for high values of cue validity because the source of misrepresentation lies in the inability to accurately estimate mutual information, which decreases as cue validity decreases (see the decreasing length of the black bars in the right panel of Fig. 1); or, the representation of conditional target entropy might be larger than the representation of simple target entropy only for low values of cue validity because the source of the misrepresentation lies in the inability to accurately partition conditional target entropy from estimates of conditional cue entropy, which increases as cue validity decreases (see the increasing length of the gray bars in the right panel of Fig. 1). Second, the representation of conditional target entropy might be overall larger than the perception of simple target entropy across the entire range of cue validity because the source of the misrepresentation reflects both types of inabilities.

The present study examined potential differences in the representation of uncertainty by adapting the demand selection paradigm originally devised to study the avoidance of cognitive demand (Kool, McGuire, Rosen, \& Botvinick, 2010). In each experiment reported in the present article, participants were offered the choice between two visual search tasks that differed only in the type and/or validity of the probability cue. Furthermore, participants selected their preferred task context explicitly at the beginning of each trial.

Potential differences in the representation of uncertainty were evaluated by comparing the observed proportion of responses with a predicted proportion, which was translated from the target entropy curves depicted in Fig. 1 by solving for the unknown proportion $x$ :

$$
\begin{gathered}
\frac{3 \text { bits }}{\text { Target entropy }}=\frac{0.50}{\text { Proportion } x} \\
\text { Proportion } x=\frac{0.50(\text { Target entropy })}{3 \text { bits }} .
\end{gathered}
$$

This equality was chosen because all but one of the experiments reported in this article used a $25 \%$ valid singledistribution probability-cue context as one of the two alternative search tasks and this search context had three bits of target entropy. Consequently, this equality was designed to return a predicted proportion of 0.50 for $x$ when the entropy of the target in the second search option was also equal to three bits, 
as in the $25 \%$ valid joint-distribution probability-cue context. Finally, the predicted proportion was obtained by taking the complement of the obtained value of $x$ :

$$
\text { Predicted proportion }=1 \text {-Proportion } x \text {. }
$$

This inversion ensured that values of target entropy that were less than three bits were associated with predicted proportions that were above chance. Figure 2 shows the resulting predicted proportions across the full range of cue and location validities.

Note that one can consider the predicted proportions in Fig. 2 to be those associated with an optimal, unbiased observer, describing what would be an ideal for a communication system in which bias plays no role. As noted by Luce (2003), "[t]he elements of choice in information theory are absolutely neutral, and lack any internal structure; the probabilities are on a pure, unstructured set whose elements are functionally interchangeable" (p. 185). Our approach is to apply information theory to reveal the conditions under which human performance deviates from that of an optimal, unbiased observer. Once those regularities are known, then it becomes possible to build models that can, in a principled way, account for those regularities.

As mentioned above and described in greater detail below, the predicted proportions in Fig. 2 play a critical role in determining the extent to which any observed preferences for single-distribution over joint-distribution probability cues was due to differences in the representation of uncertainty, as well as the nature of those differences. Given its importance, Experiment 1 was designed to provide evidence that this predicted proportion did in fact operate as intended when both of the to-be-compared search contexts involved singledistribution probability cues. With this evidence in hand, Experiment 2 directly compared participants' preferences to perform the search task within the $25 \%$ valid singledistribution probability-cue context versus within one of three separate joint-distribution probability-cue contexts of either

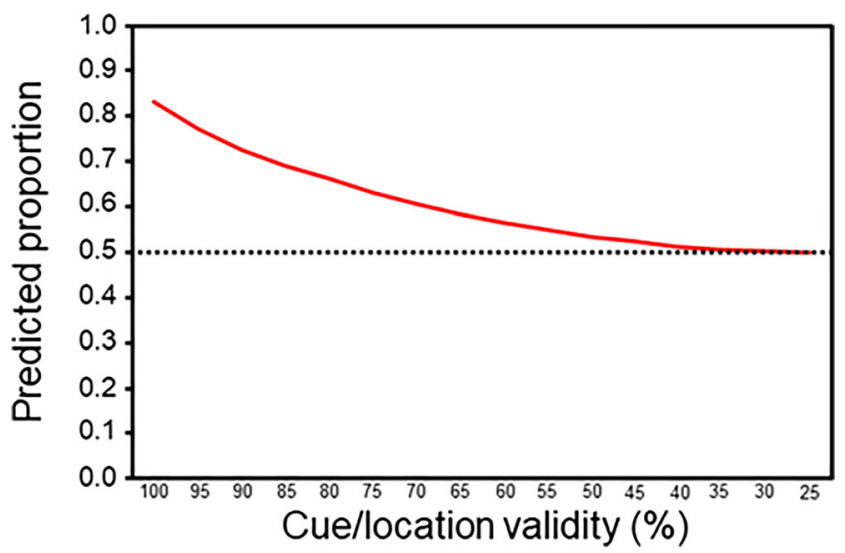

Fig. 2 Predicted proportions based on Eqs. 6 and 7 depicted as a function of cue and location validities
$100 \%, 70 \%$, or $25 \%$ (chance). This evidence suggested that the representation of conditional target entropy was overall larger and less accurate relative to the representation of simple target entropy. Experiment 3 replicated this finding within the context of an easier visual search task, and Experiment 4 confirmed this interpretation by showing that the adjusted predicted proportion, based on this newly adjusted conditional target entropy function, did in fact operate as intended when both of the to-be-compared search contexts involved jointdistribution probability cues. These experiments are summarized in Table 3.

In addition to comparing predicted and observed proportions across single- and joint-distribution probability cues, we also evaluated task performance within these different search contexts in order to consider alternative interpretations based on effort minimization (Kool et al., 2010; Pauszek \& Gibson, 2018) and/or reward maximization (Bogacz, Brown, Moehlis, Holmes, \& Cohen, 2006). If the effort minimization and/or reward maximization accounts are correct, then participants should prefer the task context that can be completed faster and/or more accurately, regardless of the uncertainty conveyed by the probability cues.

In summary, the biological imperative to minimize uncertainty was explored within the context of visual search in the present study by comparing the uncertainty associated with single-distribution and joint-distribution probability cues. Although single-distribution and joint-distribution probability cues resulted in identical objective values of target entropy, the process by which such values are obtained was found to be more complex in the context of joint-distribution probability cues relative to single-distribution probability cues. The present study therefore examined the extent to which these different methods of estimation led to differences in the representation of uncertainty, as well as the nature of such differences.

\section{Experiment 1}

Experiment 1 examined predictions in Fig. 2 by comparing two single-distribution probability-cue contexts. Participants could choose to search within the $25 \%$ valid single-distribution probability-cue context in which the target was equally likely to appear at each of four possible locations, or they could choose to search within the $70 \%$ valid single-distribution probability-cue context in which the target was $70 \%$ likely to appear at one known location (counterbalanced across participants; the target appeared randomly at the three remaining locations $10 \%$ of the time each). According to the predictions in Fig. 2, participants should prefer the $70 \%$ valid location context over the $25 \%$ valid location context, with the observed proportion averaging approximately 0.61 . 
Table 3 Summary of the four experiments reported in the present article

\begin{tabular}{|c|c|c|c|c|}
\hline Experiment & Cue context & Distractors & Subject pool & Selection Screen (Left / Right) \\
\hline 1 & $70 \% \mathrm{~S}$ vs. $25 \% \mathrm{~S}$ & Present & AMT & Blue $=70 \% \mathrm{~S} / \mathrm{Red}=25 \% \mathrm{~S}$ \\
\hline 2 & $\begin{array}{l}100 \% \text { J vs. } 25 \% \mathrm{~S} \\
70 \% \mathrm{~J} \text { vs. } 25 \% \mathrm{~S} \\
25 \% \text { J vs. } 25 \% \mathrm{~S}\end{array}$ & $\begin{array}{l}\text { Present } \\
\text { Present } \\
\text { Present }\end{array}$ & $\begin{array}{l}\text { ND } \\
\text { ND } \\
\text { AMT }\end{array}$ & $\begin{array}{l}\text { Red }=100 \% \mathrm{~J} / \text { Blue }=25 \% \mathrm{~S} \\
\text { Red }=70 \% \mathrm{~J} / \text { Blue }=25 \% \mathrm{~S} \\
\text { Blue }=25 \% \mathrm{~J} / \text { Red }=25 \% \mathrm{~S}\end{array}$ \\
\hline 3 & $\begin{array}{l}100 \% \text { J vs. } 25 \% \mathrm{~S} \\
70 \% \mathrm{~J} \text { vs. } 25 \% \mathrm{~S} \\
25 \% \text { J vs. } 25 \% \mathrm{~S}\end{array}$ & $\begin{array}{l}\text { Absent } \\
\text { Absent } \\
\text { Absent }\end{array}$ & $\begin{array}{l}\text { AMT } \\
\text { ND } \\
\text { AMT }\end{array}$ & $\begin{array}{l}\text { Blue }=100 \% \mathrm{~J} / \text { Red }=25 \% \mathrm{~S} \\
\text { Red }=70 \% \mathrm{~J} / \text { Blue }=25 \% \mathrm{~S} \\
\text { Blue }=25 \% \mathrm{~J} / \text { Red }=25 \% \mathrm{~S}\end{array}$ \\
\hline 4 & $100 \%$ J vs. $25 \% \mathrm{~J}$ & Present & AMT & Blue $=100 \% \mathrm{~J} / \mathrm{Red}=25 \% \mathrm{~J}$ \\
\hline
\end{tabular}

Note: $\mathrm{S}$ = single-distribution probability cue; $\mathrm{J}=$ joint-distribution probability cue; AMT = Amazon Mechanical Turk; ND = Notre Dame

\section{Method}

Statistical power analysis An a priori statistical power analysis was conducted for all experiments in this article using the WebPower online statistical power analysis program (Zhang $\&$ Yuan, 2015). This procedure was used to estimate the minimum total sample size needed to detect a statistically significant difference of medium effect size (Cohen's $d=0.50$ ) in the context of a one-sample, two-tailed $t$-test comparing the observed proportion to a benchmark proportion of 0.50 (indicating no preference or chance). The desired power level was set to $(1-\beta)=0.80$, and the critical alpha-level was set to $\alpha=$ 0.05 . The results of this analysis recommended a total sample size of at least 34 participants in each of the experiments reported in this study. This sample size was rounded up to 40 participants.

Participants The Institutional Review Board at the University of Notre Dame approved all procedures reported in this manuscript (protocol title: Symbolic Control of Attention; protocol number: 17-05-3920). The 40 participants in Experiment 1 were recruited through Amazon's Mechanical Turk (AMT) crowdsourcing service (www.mturk.com) in exchange for monetary payment (US\$5.00). Before participation, all of the participants were required to (1) self-report that they were a native and/or fluent English speaker, (2) self-report normal or corrected-to-normal visual acuity, (3) confirm that they were using a suitable Windows-based operating system on the computer used for testing, (4) finish the experiment with an overall percent error rate that was less than or equal to $20 \%$ (which was very easy to do if they stayed focused and remained engaged with the task), and (5) completely read and agree to the terms outlined in the informed consent document provided on the AMT webpage.

Stimuli and apparatus A web-deployable version of DMDX visual display software (Forster \& Forster, 2003) was used for all stimulus presentation and data collection. Critically, this webDMDX software automatically detects and controls for the resolutions, dimensions, and refresh rates of observers' computers during remote testing, and it has been shown to do so reliably (Witzel, Cornelius, Witzel, Forster, \& Forster, 2013).

Each trial consisted of three displays which were presented against the black background of the screen: a selection display, a fixation display, and a target display. The selection display contained two digitized images of face-down playing cards $(9.28 \mathrm{~cm}$ tall and $6.63 \mathrm{~cm}$ wide), symmetrically positioned to the left and right of central fixation and colored blue and red, respectively. The playing cards held no significance other than to provide a visible marker of the visual search task choice. The fixation display contained a small white fixation $\operatorname{dot}(0.35 \mathrm{~cm}$ in diameter $)$ in the center of the display. The target display was superimposed over the fixation display and contained a single white target letter ( $\mathrm{H}$ or $\mathrm{E}$ ) along with three non-target letters (A, P, and S). Each letter was approximately $1.51 \mathrm{~cm}$ tall and $0.75 \mathrm{~cm}$ wide, and appeared $4.37 \mathrm{~cm}$ from central fixation; the target was equally likely to appear in any of the four directions (above, below, left, or right).

Procedure Typical display sequences are depicted in the topright panel of Fig. 3. Each trial began with a selection display consisting of two colored playing cards that remained onscreen until a response was made. Kool et al. (2010) showed that variation of the appearance and location of the task markers had little to no effect on the pattern of task selections, which in their case showed a general preference for the avoidance of demand. Accordingly, in this experiment, the blue card always appeared on the left and the red card always appeared on the right (see Table 3 for a summary). Participants always pressed the "F" key with their left hand to initiate a "70\% valid location" trial or the "J" key with their right hand to initiate a "25\% valid location" trial. Participants were told that they were free to select whichever task context they preferred on each trial, provided that (1) they selected each context at least once within each block, and (2) they attempted to "make an explicit decision" on each trial, rather than making selections based on arbitrary or irrelevant criteria 
Distractor absent
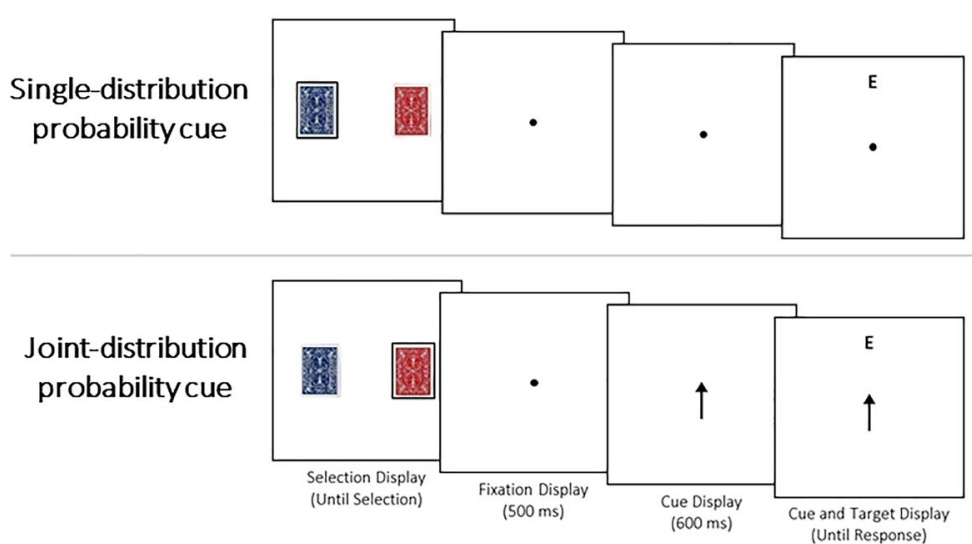

Distractor present
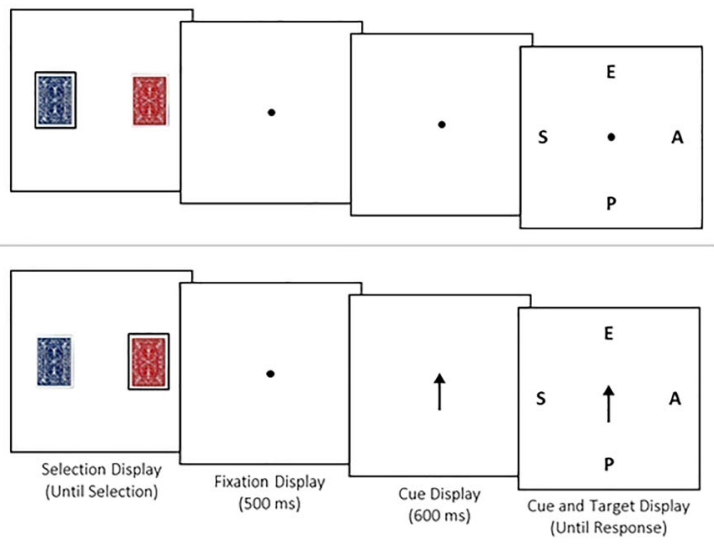

Fig. 3 Typical display sequences shown as a function of probability cue type and distractor presence

such as preferentially selecting the response button that was mapped to their dominant hand.

Immediately after this selection was made, a fixation display appeared for $1,100 \mathrm{~ms}$ followed by the target display, which remained onscreen until a response was made (or until 4,000 ms had elapsed). On each trial, the target letter was equally likely to be an $\mathrm{E}$ or $\mathrm{U}$. Observers always pressed the "F" key with their left hand to respond "E" and the "J" key with their right hand to respond "U."

There were of 640 experimental trials that were arranged into four equal blocks of 160 trials. In the $25 \%$ valid location context, the target had a $25 \%$ chance of appearing at each of the four locations in each of the four blocks, whereas in the $70 \%$ valid location context, the target had a $70 \%$ chance of appearing at one known location and it had a $10 \%$ chance of appearing at each of the three remaining locations. The most likely location was counterbalanced across participants $(n=$ 10 for each location). Participants were explicitly informed of the location validities in each of the two search contexts (cf. Geng \& Behrmann, 2005).

Before proceeding to the experimental trials, observers were required to achieve an accuracy of at least $70 \%$ in each of two practice blocks: one block of ten practice trials in the $25 \%$ valid location context, followed by a second block of ten practice trials in the $70 \%$ valid location context.

\section{Results and discussion}

Task selection The proportion of "70\% valid location" responses obtained in Experiment 1 is depicted in Fig. 4 as a function of trial block (blocks 1-4) in each of the four valid location conditions (above, below, left, and right). An initial 4 $\times 4$ mixed analysis of variance (ANOVA) with trial block as the sole within-subject factor and valid location as the sole between-subject factor. Neither the main effect of trial block,
$F(3,108)=0.14, p=.94, \eta^{2}=.004$, nor the main effect of valid location, $F(3,36)=0.03, p=.99, \eta^{2}=.003$, attained significance. Likewise, the trial block $\times$ valid location interaction also did not attain significance, $F(9,108)=1.55, p=.14$, $\eta^{2}=.11$. Consequently, we focused on the overall average proportion of " $70 \%$ valid location" responses in the remainder of this experiment.

As expected, the results suggested a preference for the $70 \%$ valid location context over the $25 \%$ valid location context in that the proportion of " $70 \%$ valid location" responses was significantly above chance $(M=0.61, S E=.04), t(39)=$ $2.68, p=.01, d=0.42$. More importantly, a $95 \%$ confidence interval (CI) computed around this average observed proportion (95\% CI: $0.53,0.69)$ included the average predicted proportion of 0.61 .

Task performance We also analyzed participants' mean correct response times (RTs) and percent error rates to discriminate the target in both search contexts. Examination of these performance measures can provide potentially important information about the extent to which the $70 \%$ valid location

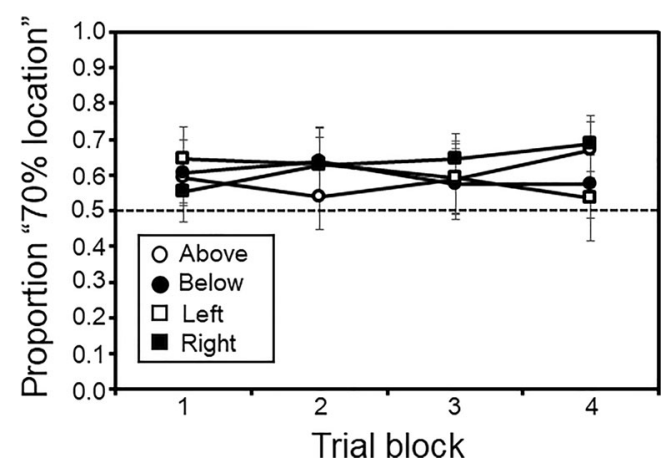

Fig. 4 The proportion of "70\% valid location" responses obtained in Experiment 1 as a function of trial block in each of the four valid location conditions. Error bars represent standard errors of the mean 
cue benefitted performance when it was selected relative to the $25 \%$ valid location context. Two planned contrasts were conducted to address these issues: the overall difference between the $70 \%$ valid location context (collapsed over spatial validity) and the $25 \%$ valid location context, and the difference between valid and invalid cue trials (i.e., the spatial cueing effect) in the $70 \%$ valid location condition.

Overall mean correct RTs were significantly faster in the $70 \%$ valid location context $(M=765 \mathrm{~ms}, S E=29.49)$ than in the $25 \%$ valid location context $(M=831 \mathrm{~ms}, S E=34.13)$, $t(39)=3.30, p=.002, d=.33$. Likewise, overall percent error rates were also significantly lower in the $70 \%$ valid location context $(M=3.06 \%, S E=0.51)$ than in the $25 \%$ valid location context $(M=4.14 \%, S E=0.71), t(39)=2.30, p=.03, d=.28$.

In addition, there was a significant spatial cueing effect observed in the $70 \%$ valid location context when mean correct RTs were analyzed. As expected, mean correct RTs were significantly faster when the target appeared at the valid location $(M=725 \mathrm{~ms}, S E=30.43)$ relative to when it appeared at one of the invalid locations $(M=844 \mathrm{~ms}, S E=28.45), t(39)=$ $6.11, p<.001, d=.63$. Likewise, percent error rates were significantly lower when the target appeared at the valid location $(M=2.64 \%, S E=0.50)$ relative to when it appeared at one of the invalid locations $(M=4.08 \%, S E=0.61), t(39)=$ $3.74, p=.001, d=.41$. These task-performance results are discussed further in the General discussion.

In summary, the task selection results of Experiment 1 provided some evidence for the validity of the predicted proportions (Fig. 2) that were translated from the target entropy curves (Fig. 1), at least when both of the to-be-compared search alternatives involved single-distribution probability cues. Although limited in scope, this finding provides some evidence that good correspondence can be achieved between the predicted and observed proportions without including other potential sources of bias in the prediction.

\section{Experiment 2}

In Experiment 2, participants were given the choice between single-distribution and joint-distribution probability-cue contexts (see Table 3 for a summary). In particular, participants could choose to search within the $25 \%$ valid location context in which the target was equally likely to appear at each of four possible locations (as in Experiment 1); or, they could choose to search within one of three joint-distribution probability cue conditions (assessed within separate groups): $100 \%$ valid, $70 \%$ valid, or $25 \%$ valid cue contexts. According to the predicted proportions in Fig. 2, participants should prefer the $100 \%$ valid and $70 \%$ valid (joint-distribution) cue contexts over the $25 \%$ valid (single-distribution) location cue context; however, they should have no preference when choosing between the $25 \%$ valid cue context and the $25 \%$ valid location context.

Furthermore, two potential patterns of differences between the observed and predicted proportions were of particular interest in Experiment 2. First, the observed proportion of "jointdistribution cue" responses might be smaller than the predicted proportion over a limited range of cue validity values, perhaps because of a limited ability to estimate mutual information or a limited ability to partition conditional cue entropy. Second, the observed proportion of "joint-distribution cue" responses might be consistently smaller than the predicted proportion across the entire range of cue validity.

\section{Method}

Participants A total of 120 individuals were recruited to participate in Experiment 2, with 40 different individuals assigned to each of the three conditions. The participants were recruited from two sources. The three cue validity conditions reported in Experiment 2 were originally intended to be reported as independent experiments and there was no attempt to control for recruitment source across the three cue validity conditions. The experiments are reported together for the sake of brevity. Participants in the $100 \%$ valid cue context and the $70 \%$ valid cue context were recruited from the undergraduate subject pool at the University of Notre Dame in exchange for course credit, whereas participants in the $25 \%$ valid cue context were recruited from AMT in exchange for US\$5.00. The subject restrictions that were imposed on the undergraduate participants were the same as those imposed on the AMT participants.

Stimuli and apparatus The stimuli and apparatus used for the AMT experiment were identical to those used in Experiment 1. The stimuli and apparatus were identical for the undergraduate participants with the following exceptions. First, a personal desktop computer running DMDX display software (Forster \& Forster, 2003) with a 22-in. LCD monitor was used for all stimulus presentation and data collection. Second, responses were collected using either a custom-built response box or standard computer keyboard with response times rounded to the nearest millisecond. Third, a fixed viewing distance of $57 \mathrm{~cm}$ was enforced using a chin-rest and the experiment was conducted in a dimly lit room. Despite these differences, we did not consider testing environment to be important because there have been several findings from laboratory-based studies of visual attention which have been successfully replicated on AMT with good fidelity (see, e.g., Crump, McDonnell, \& Gureckis, 2013), including our own studies using the spatial cueing paradigm (Pauszek, Sztybel, \& Gibson, 2017), suggesting that this difference in task environment exerts little to no effect on the quality or interpretability of obtained results. 
Regardless of testing environment, the three displays used in Experiment 2 were identical to the three displays used in Experiment 1, with the sole exception being that a cue display was inserted between the fixation and target displays in the three joint-distribution probability-cue contexts. The cue display contained an arrow cue $(\uparrow, \downarrow, \leftarrow$, or $\rightarrow)$ that was presented in white and that varied in height and width from $0.48 \mathrm{~cm}$ to $1.18 \mathrm{~cm}$ depending on the direction it was pointing.

Procedure The procedure used in Experiment 2 was identical to the procedure used in Experiment 1 with the sole exception being that observers chose between single-distribution and joint-distribution probability-cue contexts in the present experiment. Typical display sequences in each of the singledistribution and joint-distribution probability-cue contexts are depicted in the top-right and bottom-right panels of Fig. 3 , respectively. Each trial began with a selection display consisting of two colored playing cards that remained onscreen until a response was made. Kool et al. (2010) showed that variation of the appearance and location of the task markers had little to no effect on the pattern of task selections. In the "AMT" version of the experiment, the blue card always appeared on the left and the red card always appeared on the right, whereas in the "Notre Dame" version of the experiment, the red card always appeared on the left and the blue card always appeared on the right (see Table 3 for a summary). Regardless, participants always pressed the "F" key with their left hand to initiate a "joint-distribution cue" trial or the "J" key with their right hand to initiate a "singledistribution cue" trial. Immediately after a selection was made, a fixation display appeared for $500 \mathrm{~ms}$. On "single-distribution cue" trials, the fixation display remained onscreen for an additional $600 \mathrm{~ms}$; however, on "joint-distribution cue" trials, the fixation dot was replaced by a cue display, and this also remained onscreen for $600 \mathrm{~ms}$. Following either 600 -ms duration, the target display was superimposed on the fixation or cue display and remained onscreen until a response was made (or until 4,000 ms had elapsed).

Participants' task was to discriminate the identity of the target letter (E or $\mathrm{U}$ ) as quickly and as accurately as possible. As in Experiment 1, participants always pressed the "F" key with their left hand to respond "E" and the "J" key with their right hand to respond "U." Gibson and Kingstone (2005) examined potential interactions between the orientation of the response pad and the orientation of the cue, but they failed to find evidence of either facilitation or interference when, for example, observers oriented in the left cued direction and responded using the left or right key, respectively, relative to using the top or bottom key (see also Gibson \& Davis, 2011).

There were 640 experimental trials that were arranged into four equal blocks of 160 trials. In the single-distribution cue context, the target had a $25 \%$ chance of appearing at each of the four locations in each of the four blocks, whereas in the joint-distribution cue context, the target had either a $100 \%$, $70 \%$, or $25 \%$ chance of appearing at the cued location on valid trials, and it had an equal chance of appearing at one of the three remaining uncued locations on invalid trials (when cue validity was less than $100 \%$ ). Participants were explicitly informed of the appropriate location and cue validities in each of the three joint-distribution probability-cue contexts.

Before proceeding to the experimental trials, observers were required to achieve an accuracy of at least $70 \%$ in each of two practice blocks: one block of ten practice trials in the single-distribution probability-cue context (in which location was $25 \%$ valid), followed by a second block of ten practice trials in which the arrow cue was $100 \%, 70 \%$, or $25 \%$ valid.

\section{Results and discussion}

Task selection The proportion of "joint-distribution cue" responses obtained in Experiment 2 is depicted in Fig. 5 as a function of trial block (blocks 1-4) in each of the three jointdistribution valid cue contexts $(100 \%, 70 \%$, and $25 \%)$. An initial $4 \times 3$ mixed ANOVA was conducted to determine the extent to which the proportion of "joint-distribution cue" responses varied across the four trial blocks - a within-subject

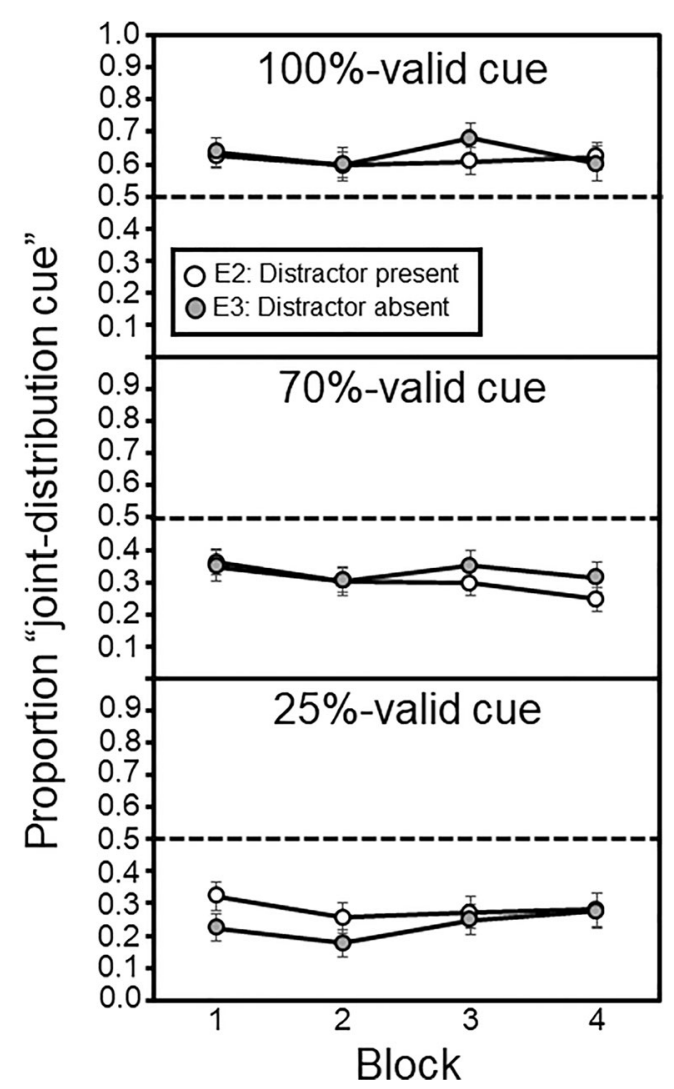

Fig. 5 The proportion of "joint-distribution cue" responses obtained in Experiments 2 and 3 as a function of trial block in each of the three jointdistribution cue contexts. Error bars represent standard errors of the mean 
factor - in each of the three joint-distribution valid cue contexts $-\mathrm{a}$ between-subject factor. There were significant main effects of trial block, $F(3,351)=3.41, p=.02, \eta^{2}=.03$, and joint-distribution cue context, $F(2,117)=24.98, p<.001, \eta^{2}$ $=.30$; however, the two-way interaction between these two variables did not attain significance, $F(6,351)=1.23, p=.29$, $\eta^{2}=.02$. The main effect of joint-distribution cue context are discussed in greater below. The main effect of trial block reflected a slightly higher proportion of "joint-distribution cue" responses in the first block of trials $(M=0.44)$ relative to the other three blocks ( $M=0.39$ block $2 ; M=0.39$ block 3 ; and, $M=0.38$ block 4); however, none of the post hoc Bonferroni-corrected pairwise comparisons (with the critical alpha value equal to $0.05 / 3=0.017$ ) attained significance. Furthermore, the pattern of "joint-distribution cue" responses remained consistently above chance (as in the $100 \%$ valid cue context) or below chance (as in the $70 \%$ valid and $25 \%$ valid cue contexts) across trial block. Hence, we focused on the overall average proportion of "joint-distribution cue" responses in the remainder of this section.

The average proportions of observed and predicted "jointdistribution cue" responses are depicted in the upper panel of Fig. 6 as a function of cue validity context. As expected, the pattern of findings suggested a preference for the $100 \%$ valid cue context over the $25 \%$ valid location context in that the proportion of "joint-distribution cue" responses was

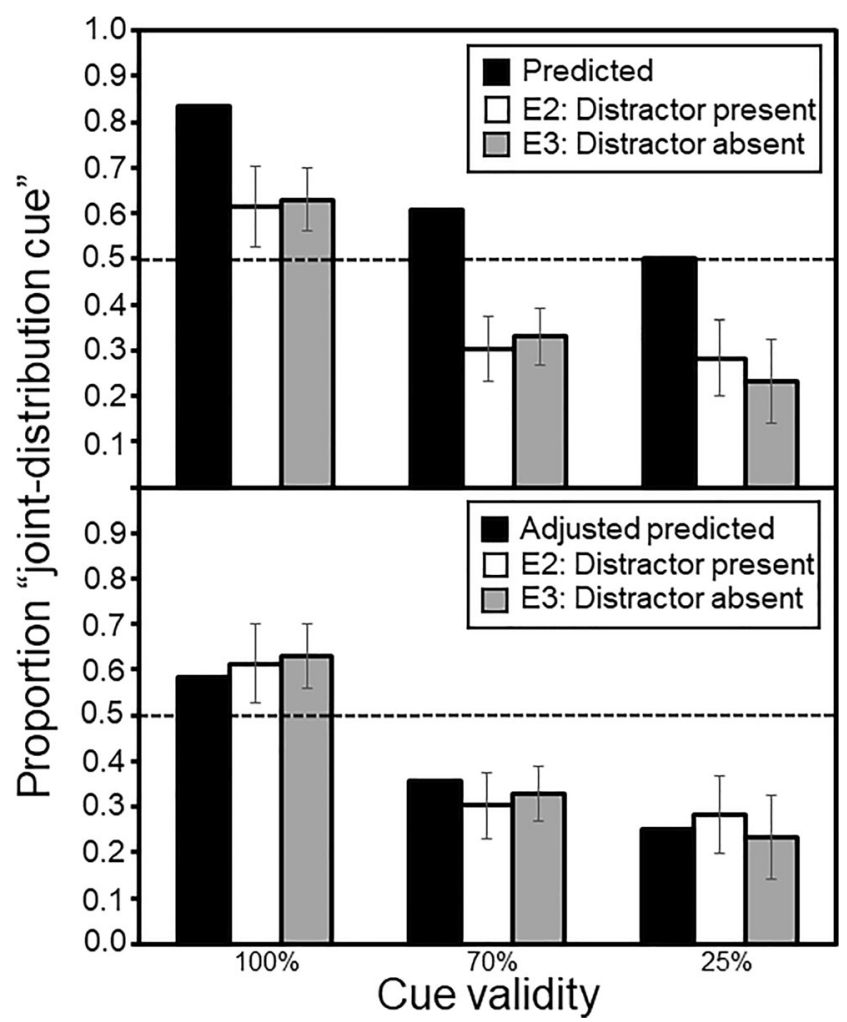

Fig. 6 Proportions of predicted and observed "joint-distribution cue" responses shown as a function of cue validity in Experiments 2 and 3. Error bars represent $95 \%$ confidence intervals significantly above chance in the $100 \%$ valid cue context $(M$ $=0.61, S E=.03), t(39)=3.33, p=.002, d=0.53$. Surprisingly, the pattern of findings suggested a preference for the $25 \%$ valid location context over the $70 \%$ valid and $25 \%$ valid cue contexts in that the proportion of "joint-distribution cue" responses was significantly below chance in the $70 \%$ valid cue context $(M=0.30, S E=.03), t(39)=6.56, p<$ $.001, d=1.04$; and in the $25 \%$ valid cue context $(M=0.28, S E$ $=.05), t(39)=4.77, p<.001, d=0.75$.

Moreover, it is worth pointing out that the observed proportion of " $70 \%$ valid cue" responses obtained in Experiment 2 was significantly less than the observed proportion of " $70 \%$ valid location" responses obtained in Experiment $1(M=0.61$, $S E=.04), t(78)=6.09, p<.001, d=1.36$, even though both $70 \%$ valid contexts were compared against the same $25 \%$ valid single-distribution probability-cue context. This finding is also important because it mitigates concerns that the lowerthan-expected observed proportions observed across the three joint-distribution cue contexts in Experiment 2 may have occurred because participants were biased to press the "right" key (which was consistently associated with the $25 \%$ valid single-distribution probability cue) more than the "left" key (which was consistently associated with each of the three joint-distribution cues) during the selection screen. Had this been the case, then a similar pattern of lower-than-expected observed proportions should have also been found in Experiment 1, which did not occur.

Task performance Overall mean correct RTs and percent error rates are shown in Fig. 7 as a function of cue type (jointdistribution cue vs. single-distribution cue) in each of the three joint-distribution cue contexts (100\% vs. $70 \%$ vs. $25 \%)$. A $2 \times$ 3 mixed ANOVA was conducted on mean correct RTs and percent error rates with cue type as the sole within-subject factor and joint-distribution cue context as the sole betweensubject factor. With respect to mean correct RT, there were significant main effects of cue type, $F(1,117)=16.54, p<$ $.001, \eta^{2}=.12$, and joint-distribution cue context, $F(2,117)=$ $7.86, p<.001, \eta^{2}=.12$. In addition, the interaction between these variables was also significant, $F(2,117)=25.08, p<$ $.001, \eta^{2}=.30$. With respect to percent error rates, there was a significant main effect of joint-distribution cue context, $F(2$, $117)=3.44, p=.04, \eta^{2}=.06$, but neither the main effect of cue type, $F(1,117)=0.06, p=.82, \eta^{2}=.00$, nor the interaction, $F(2,117)=2.31, p=.10, \eta^{2}=.04$, was found to be significant.

As can be seen in Fig. 7, the significant two-way interaction between cue type and joint-distribution cue context that was observed with mean correct RTs reflected the fact that performance was significantly faster in the $100 \%$ valid cue context $(M=525 \mathrm{~ms}, S E=36.78)$ than in the $25 \%$ valid location context $(M=700 \mathrm{~ms}, S E=42.77), t(39)=8.27, p<$ $.001, d=.69$. However, mean correct RTs did not differ 


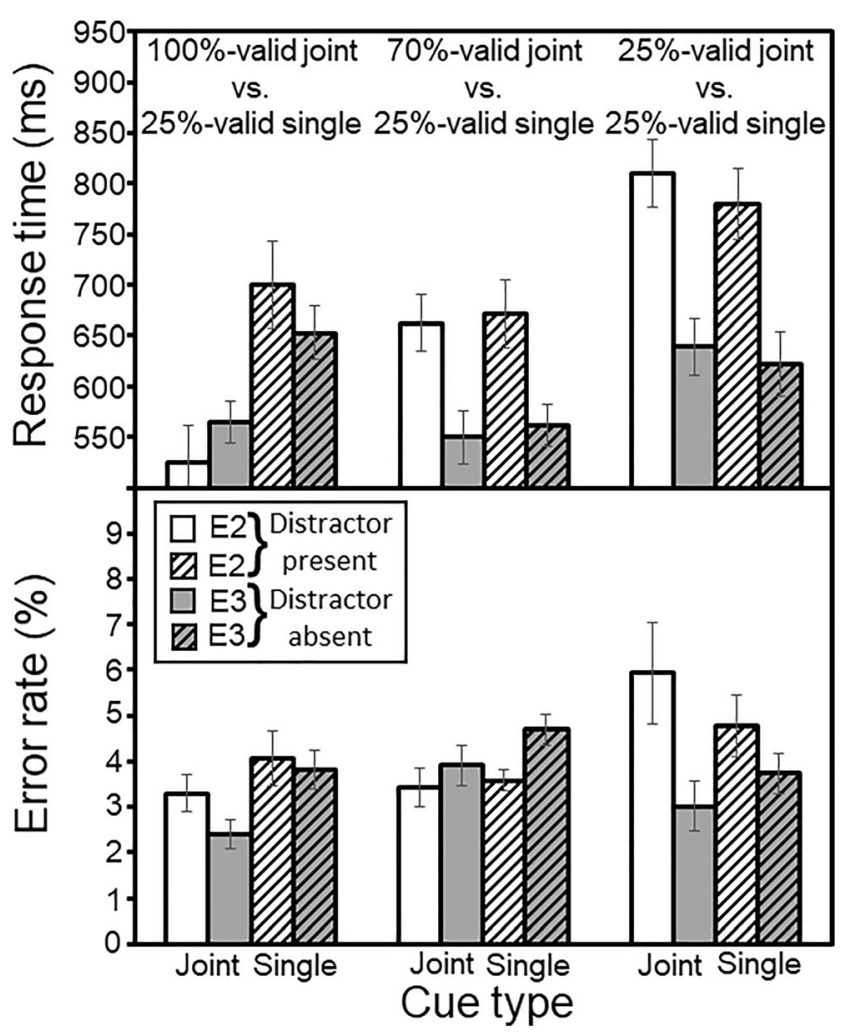

Fig. 7 Mean correct response times and percent error rates obtained in Experiments 2 and 3 as a function of cue type in each of the three jointdistribution cue contexts. Error bars represent standard errors of the mean

significantly as a function of joint-distribution cue versus single-distribution cue in the $70 \%$ valid cue context $(M=$ $662 \mathrm{~ms}, S E=27.95$ vs. $M=671 \mathrm{~ms}, S E=33.44$, respectively, $t(39)=0.52, p=.61, d=.05)$; or, in the $25 \%$ valid cue context $(M=810 \mathrm{~ms}, S E=33.61$ vs. $M=780 \mathrm{~ms}, S E=34.64$, respectively, $t(39)=1.16, p=.25, d=.14)$. Identical analyses conducted on the percent error rates across the three jointdistribution cue conditions produced similar results to those obtained with mean correct RTs $(p=.10, p=.72$, and $p=.22$, respectively). These task-performance results are discussed further in the General discussion.

The finding that overall performance did not benefit from the cue in the $70 \%$ valid cue context may suggest that participants did not use these cues to guide their search. However, as can be seen in Fig. 8, this was not the case: mean correct RTs were significantly faster on valid trials $(M=590 \mathrm{~ms}, S E=$ 26.99) than on invalid cue trials $(M=813 \mathrm{~ms}, S E=34.28)$, $t(39)=8.90, p<.001, d=1.14$; and percent error rates were significantly lower on valid trials $(M=3.03 \%, S E=0.45)$ than on invalid trials $(M=5.28 \%, S E=0.26), t(39)=2.03, p=.05$, $d=.38$.

A similar pattern was also observed in the $25 \%$ valid cue context (see Fig. 8; note that one participant was excluded from this analysis because of missing data; in particular, this participant failed to encounter any valid trials due to the low likelihood of these trials coupled with a low selection

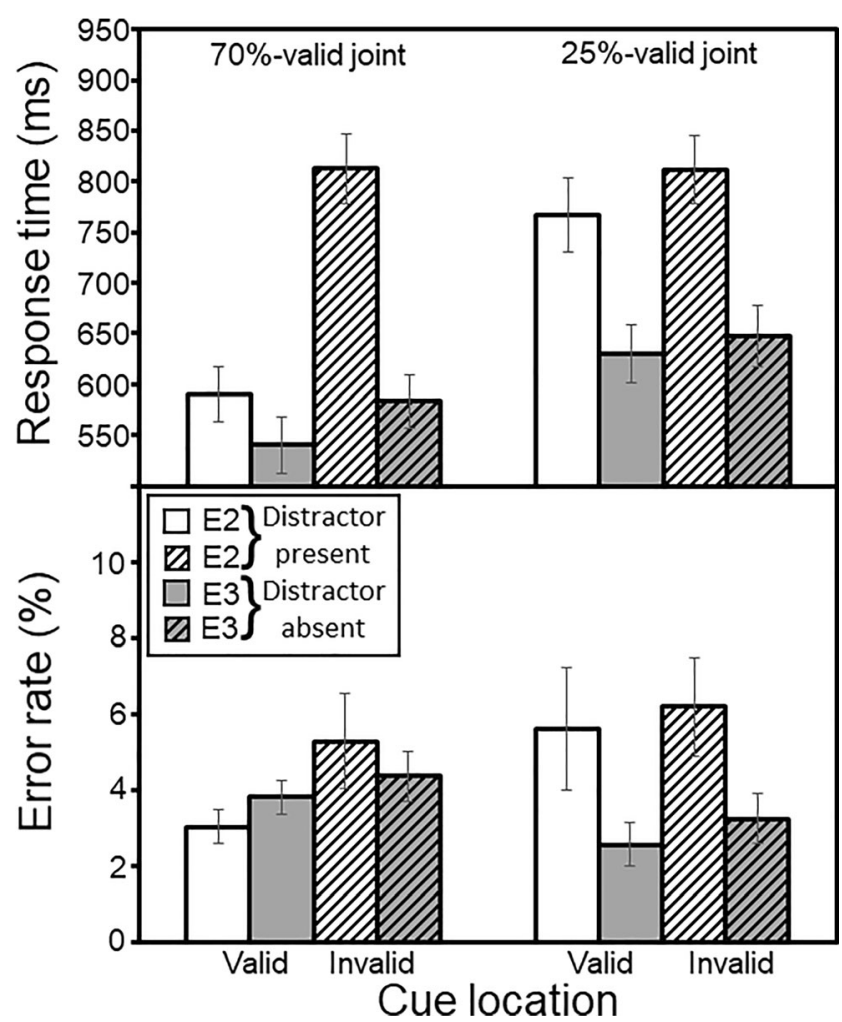

Fig. 8 Mean correct response times and percent error rates obtained in Experiments 2 and 3 as a function of cue location in each of the $70 \%$ valid and $25 \%$ valid cue contexts. Error bars represent standard errors of the mean

proportion). The results indicated that mean correct RTs were significantly faster on valid cue trials $(M=767 \mathrm{~ms}, S E=$ 37.30) than on invalid cue trials $(M=812 \mathrm{~ms}, S E=33.13)$, $t(38)=2.49, p=.02, d=.20$; however, the difference between valid and invalid cue trials was not significant when percent error rates were analyzed $(M=5.61 \%, S E=1.63$ vs. $M=$ $6.18 \%, S E=1.28$, respectively), $t(38)=0.31, p=.76, d=.06$.

Moreover, a $2 \times 2$ mixed ANOVA with cue location (valid vs. invalid) as the sole within-subject factor and jointdistribution cue context (70\% vs. $25 \%$ ) as the sole betweensubject factor indicated that the 222-ms spatial cueing effect (invalid RT - valid RT) observed in the $70 \%$ valid cue context was significantly larger than the 45 -ms spatial cueing effect observed in the $25 \%$ valid cue context, $F(1,77)=32.60, p<$ $.001, \eta^{2}=.30$, for the cue location $\times$ joint-distribution cue context interaction. The same interaction did not attain significance when percent error rates were analyzed, $F(1,77)=$ $0.62, p=.43, \eta^{2}=.01$.

In summary, comparison of the observed and predicted proportions of "joint-distribution cue" responses in the task selection results indicated that the average observed proportions were consistently lower than the predicted proportions. In fact, as can be seen in the upper panel of Fig. 6, none of the 95\% CIs computed around the three average observed proportions included the corresponding predicted proportion. This 
finding is important and suggests that participants choose the joint-distribution probability cues less than expected, perhaps because the uncertainty these cues conveyed about the identity and location of the target was represented less accurately than for single-distribution probability cues. However, before interpreting this pattern of findings further, we first attempted to replicate it in the next experiment.

\section{Experiment 3}

Experiment 3 was identical to Experiment 2, with the sole exception being that the target letter was the sole letter presented in the target display (see the two left-hand panels in Fig. 3). The absence of distractors was expected to increase the efficiency of finding the target in the absence of any jointdistribution probability cue; however, it had no impact on the various entropy values shown in Fig. 1. Thus, the distractor manipulation was potentially useful in assessing the extent to which other factors besides target entropy might influence task choice. To the extent that the pattern of task choices observed in Experiment 2 were influenced primarily by estimates of target entropy, the same pattern should be observed in Experiment 3.

\section{Method}

Participants A total of 120 individuals were recruited to participate in Experiment 3, with 40 different individuals assigned to each of the three conditions. The participants were recruited from two sources. The three cue validity conditions reported in Experiment 3 were originally intended to be reported as independent experiments and there was no attempt to control for recruitment source across the three cue validity conditions. The experiments are reported together for the sake of brevity. Participants in the $70 \%$ valid cue context were recruited from the undergraduate subject pool at the University of Notre Dame in exchange for course credit, whereas participants in the $100 \%$ valid cue context and the $25 \%$ valid cue context were recruited through AMT in exchange for US\$5.00. The subject restrictions that were imposed on the undergraduate participants were the same as those imposed on the AMT participants (see Table 3 for a summary).

Stimuli and apparatus The stimuli and apparatus used in Experiment 2 were identical to those used in Experiment 3, with the sole exception being that the non-target distractor letters were removed from the target display.

Procedure The procedure used in Experiment 3 was identical to the procedure used in Experiment 2. Typical display sequences in the single-distribution and joint-distribution probability-cue contexts are depicted in the top-left and bottom-left panels of Fig. 3, respectively (see Table 3 for a summary).

\section{Results and discussion}

Task selection The proportion of "joint-distribution cue" responses obtained in Experiment 3 is depicted in Fig. 5 as a function of trial block (blocks 1-4) in each of the three jointdistribution valid cue contexts $(100 \%, 70 \%$, and $25 \%)$. An initial $4 \times 3$ mixed ANOVA was run to determine the extent to which the proportion of "joint-distribution cue" responses varied across the four trial blocks - a within-subject factor - in each of the three joint-distribution valid cue contexts - a between-subject factor. There were significant main effects of trial block, $F(3,351)=2.72, p=.04, \eta^{2}=.02$, and jointdistribution cue context, $F(2,117)=26.46, p<.001, \eta^{2}=.31$; however, the two-way interaction between these two variables did not attain significance, $F(6,351)=0.88, p=.51, \eta^{2}=.02$. The main effect of joint-distribution cue context are discussed in greater below. The main effect of trial block reflected a slightly lower proportion of "joint-distribution cue" responses in the second block of trials $(M=0.36)$ relative to the other three blocks $(M=0.40$ block $1 ; M=0.43$ block 3 ; and, $M=$ 0.40 block 4 ); however, only the difference between block 2 and block 3 was found to be significant following Bonferroni correction (with the critical alpha value equal to $0.05 / 3=$ $0.017), t(119)=3.18, p=.002, d=.20$. Nevertheless, the pattern of "joint-distribution cue" responses remained consistently above chance (as in the $100 \%$ valid cue context) or below chance (as in the $70 \%$ valid and $25 \%$ valid cue contexts) across trial block. Hence, we focused on the overall average proportion of "joint-distribution cue" responses in the remainder of this section.

The proportions of observed and predicted "joint-distribution cue" responses are depicted in the upper panel of Fig. 6 as a function of cue validity context. Consistent with Experiment 2 , the pattern of findings suggested a preference for the $100 \%$ valid cue context over the $25 \%$ valid location context in that the proportion of "joint-distribution cue" responses was significantly above chance in the $100 \%$ valid cue context $(M=$ $0.63, S E=.04), t(39)=2.98, p=.005, d=1.03$. Also consistent with Experiment 2, the pattern of findings suggested a preference for the $25 \%$ valid location context over the $70 \%$ valid and $25 \%$ valid cue contexts in that the proportion of "joint-distribution cue" responses was significantly below chance in the $70 \%$ valid cue context $(M=0.33, S E=.04)$, $t(39)=4.78, p<.001, d=0.76$; and in the $25 \%$ valid cue context $(M=0.23, S E=.04), t(39)=6.49, p<.001, d=1.03$. Furthermore, comparison of the observed and predicted proportions of "joint-distribution cue" responses indicated that the average observed proportions were consistently lower than the predicted proportions, and none of the $95 \%$ CIs computed 
around the three average observed means included the corresponding predicted proportion (see upper panel of Fig. 6).

The similar pattern of results observed across Experiments 2 and 3 was also evaluated within the context of a 3 (cue validity context: $100 \%$ valid vs. $70 \%$ valid vs. $25 \%$ valid $) \times$ 2 (distractor presence: present vs. absent) independent-groups ANOVA. This analysis revealed a main effect of cue validity context, $F(2,234)=50.97, p<.001, \eta^{2}=.30$. Bonferronicorrected post hoc tests indicated that the proportion of "joint-distribution cue" responses was significantly higher in the $100 \%$ valid cue context condition $(M=0.62, S E=.03)$ than in the $70 \%$ valid cue context $(M=0.32, S E=.02), t(158)$ $=8.48, p<.001, d=1.34$; and in the $25 \%$ valid cue context $(M=0.26, S E=.03), t(158)=8.48, p<.001, d=1.40$. However, the difference between the $70 \%$ valid cue and $25 \%$ valid cue contexts did not attain significance, $t(158)=$ $1.54, p=.13, d=0.24$. Neither the main effect of distractor presence, $F(1,234)=0.01, p=.93, \eta^{2}=.00$, nor the interaction between cue validity context and distractor presence attained significance, $F(2,234)=0.59, p=.56, \eta^{2}=.005$. Thus, the pattern of findings obtained in Experiment 3 corroborated the pattern obtained in Experiment 2, despite the difference in distractor presence (or display size).

In addition, it is also worth pointing out that the similar pattern of results observed in the $100 \%$ valid cue context of Experiments 2 and 3 also mitigates concerns about potential differences in two subject pools, as the participants were recruited from the University of Notre Dame in Experiment 2 and they were recruited from AMT in Experiment 3. Moreover, this similar pattern of results also mitigates concerns about the color of the two task markers (i.e., playing cards) displayed in the initial task selection screen, as the task marker for the $100 \%$ valid cue context was red in Experiment 2 and it was blue in Experiment 3.

In order to determine the extent to which the difference between observed and predicted proportions varied across cue validity context, the predicted proportion was subtracted from each participant's observed proportion, and an identical $3 \times 2$ independent-groups ANOVA was conducted on these difference scores. As expected, neither the main effect of distractor presence, nor the interaction between cue validity context and distractor presence was significant (both $F \mathrm{~s}<1$ ). Of most critical importance, the main effect of cue validity context was not significant either, $F(2,234)=2.12, p=.12$, $\eta^{2}=.02$; rather, the difference score remained relatively constant across cue validity context, attaining an average value of $-0.25(S E=0.02)$.

Task performance Mean correct RTs and percent error rates are shown in Fig. 7 as a function of cue type (joint-distribution cue vs. single-distribution cue) in each of the three jointdistribution cue contexts $(100 \%$ vs. $70 \%$ vs. $25 \%)$. A $2 \times 3$ mixed ANOVA was conducted on mean correct RTs and percent error rates with cue type as the sole within-subject factor and joint-distribution cue context as the sole betweensubject factor. With respect to mean correct RTs, there was a significant main effect of cue type, $F(1,117)=8.71, p=.004$, $\eta^{2}=.07$; the main effect of joint-distribution cue context did not attain significance, $F(2,117)=2.46, p=.09, \eta^{2}=.04$. In addition, the interaction between these variables was also significant, $F(2,117)=11.54, p<.001, \eta^{2}=.16$. With respect to percent error rates, there was a significant main effect of cue type, $F(1,117)=14.15, p<.001, \eta^{2}=.11$, but neither the main effect of joint-distribution cue context, $F(2,117)=2.97$, $p=.06, \eta^{2}=.05$, nor the interaction, $F(2,117)=0.75, p=.47$ ,$\eta^{2}=.01$, was found to be significant.

As can be seen in Fig. 7, the significant two-way interaction between cue type and joint-distribution cue context that was observed with mean correct RTs reflected the fact that performance was significantly faster in the $100 \%$ valid cue context $(M=564 \mathrm{~ms}, S E=20.69)$ than in the $25 \%$ valid location context $(M=653 \mathrm{~ms}, S E=26.03), t(39)=5.10, p<$ $.001, d=.60$. However, mean correct RTs did not differ significantly as a function of joint-distribution cue versus singledistribution cue in the $70 \%$ valid cue context $(M=550 \mathrm{~ms}, S E$ $=26.39$ vs. $M=561 \mathrm{~ms}, S E=20.49$, respectively, $t(39)=$ $0.64, p=.52, d=.07)$; or, in the $25 \%$ valid cue context $(M=$ $639 \mathrm{~ms}, S E=28.13$ vs. $M=622 \mathrm{~ms}, S E=31.76$, respectively, $t(39)=1.13, p=.27, d=.09)$. Identical analyses conducted on the percent error rates across the three joint-distribution cue conditions produced similar results to those obtained with mean correct RTs ( $p=.001, p=.04$, and $p=.19$, respectively). These task-performance results are discussed further in the General discussion.

The finding that performance did not benefit from the cue in the $70 \%$ valid cue context may suggest that participants did not use these cues to guide their search. However, as can be seen in Fig. 8, this was not the case: Mean correct RTs were significantly faster on valid cue trials $(M=540 \mathrm{~ms}, S E=$ 28.23) than on invalid cue trials $(M=583 \mathrm{~ms}, S E=26.79)$, $t(39)=4.57, p<.001, d=.25$; the corresponding comparison of percent error rates revealed no significant difference between valid trials $(M=3.80 \%, S E=0.43)$ and invalid trials $(M=4.36 \%, S E=0.67), t(39)=1.00, p=.32, d=.16$.

A similar pattern was also observed in the $25 \%$ valid cue context (see Fig. 8; note that two participants were excluded from this analysis because of missing data; in particular, these two participants failed to encounter any valid trials due to the low likelihood of these trials coupled with a low selection proportion). However, the numerical difference observed between the mean correct RTs on valid cue $(M=630 \mathrm{~ms}, S E=29.39)$ and invalid cue $(M=648 \mathrm{~ms}, S E=30.24)$ trials did not attain significance in this experiment, $t(37)=1.36, p=.18, d=.10$. Likewise, the numerical difference observed between percent error rates on valid $(M=2.56 \%, S E=0.56)$ and 
invalid $(M=3.24 \%, S E=0.66)$ trials also did not attain significance in this experiment, $t(37)=0.88, p=.39, d=$ .18 .

Moreover, although the 44-ms spatial cueing effect observed in the $70 \%$ valid cue context was numerically larger than the 17 -ms spatial cueing effect observed in the $25 \%$ valid cue context, this difference did not attain significance, $F(1$, $76)=2.79, p=.10, \eta^{2}=.04$, for the cue location (valid vs. invalid) $\times$ joint-distribution cue context $(70 \%$ valid vs. $25 \%$ valid) interaction. The same interaction also did not attain significance when percent error rates were analyzed, $F(1$, 76) $=0.02, p=.90, \eta^{2}=.00$.

We also conducted an additional analysis that compared overall task performance across Experiments 2 and 3 to evaluate the extent to which the presence versus absence of distractors influenced performance. In the first analysis, a 2 $\times 2 \times 3$ mixed ANOVA was conducted on mean correct RTs and percent error rates, with cue type (joint-distribution cue vs. single-distribution cue) as the sole within-subject factor, and with distractor presence (E2: present vs. E3: absent) and joint-distribution cue context (100\% vs. $70 \%$ vs. $25 \%)$ as the two between-subject factors. To streamline the discussion, we focused exclusively on the main effect and interactions involving distractor presence.

With respect to mean correct RTs, there was a significant main effect of distractor presence, $F(1,234)=15.15, p<.001$, $\eta^{2}=.06$, as well as a significant two-way interaction between joint-distribution cue context and distractor presence, $F(2$, 234) $=3.84, p=.02, \eta^{2}=.03$, and a significant three-way interaction between cue type, joint-distribution cue context, and distractor presence, $F(2,234)=4.01, p=.02, \eta^{2}=.03$. Of the six possible distractor present versus distractor absent comparisons that could be conducted across the 2 cue type $\times 3$ joint-distribution cue context conditions, mean correct RTs were significantly faster in the distractor absent condition than in the distractor present condition in all conditions (all $p \mathrm{~s}=$ .006 or less), except those in the $100 \%$ valid cue versus $25 \%$ valid location contexts ( $p=.36$ and $p=.35$, respectively). The null effect of distractor presence in these two conditions may reflect differences in the subject populations as participants recruited from an undergraduate population served in the distractor present version of the experiment whereas participants recruited from the AMT population served in the distractor absent version of the experiment (see Table 3 for a summary). Nevertheless, the results generally supported the notion that the search task could be performed more efficiently in the distractor absent condition than in the distractor present condition.

With respect to percent error rates, there were significant two-way interactions between joint-distribution cue context and distractor presence, $F(2,234)=4.42, p=.01, \eta^{2}=.04$, and between cue type and distractor presence, $F(1,234)=$ $5.45, p=.02, \eta^{2}=.02$. For the former interaction, percent error rates were significantly lower in the distractor absent condition relative to the distractor present condition when the joint-distribution cue context was $25 \%$ ( $p=.03$ ), but not in the other two joint-distribution cue contexts (both $p \mathrm{~s}=.07$ or greater). For the latter interaction, percent error rates were significantly lower in the distractor absent condition relative to the distractor present condition when a joint-distribution probability cue was shown $(p=.03)$, but not when the singledistribution (25\% valid) probability cue was shown $(p=.89)$.

In summary, the pattern of task selection results observed in Experiment 3 was very similar to the pattern observed in Experiment 2. Most importantly, the main results showed that the observed proportions of "joint-distribution cue" responses were on average 0.25 less than the corresponding predicted proportions. This difference can be interpreted to suggest that the representation of conditional target entropy (associated with joint-distribution cues) might be overall larger and less accurate across the range of probability cues relative to the representation of simple target entropy (associated with single-distribution cues). We estimated this "adjusted" conditional target entropy curve by first subtracting the adjusted predicted proportion (i.e., the original predicted proportion 0.25 ) from one. We then estimated adjusted target entropy by solving for the unknown target entropy $x$ :

$$
\begin{aligned}
\frac{0.50}{\text { Adjusted proportion }} & =\frac{3 \text { bits }}{\text { Adjusted target entropyx }} \\
\text { Adjusted target entropy } x & =\frac{3 \text { bits }(\text { Adjusted proportion })}{0.50} .
\end{aligned}
$$

The adjusted conditional target entropy curve can be seen in the upper panel of Fig. 9, and the resulting adjusted predicted proportions (using Eqs. 6 and 7) can be seen in the lower panel of Fig. 9 along with the original predicted proportion. The proportions of observed and adjusted predicted "jointdistribution cue" responses are depicted in the lower panel of Fig. 6 as a function of cue validity context; all six of the observed 95\% CIs obtained from both Experiments 2 and 3 now included the corresponding adjusted predicted proportion. This finding suggests that the representation of uncertainty was overall greater for joint-distribution versus singledistribution probability cues, as shown in the upper panel of Fig. 9.

\section{Experiment 4}

Experiment 4 was conducted to provide stronger support for the notion that the representation of uncertainty was overall greater for joint-distribution versus single-distribution probability cues, as suggested by the adjusted conditional target 


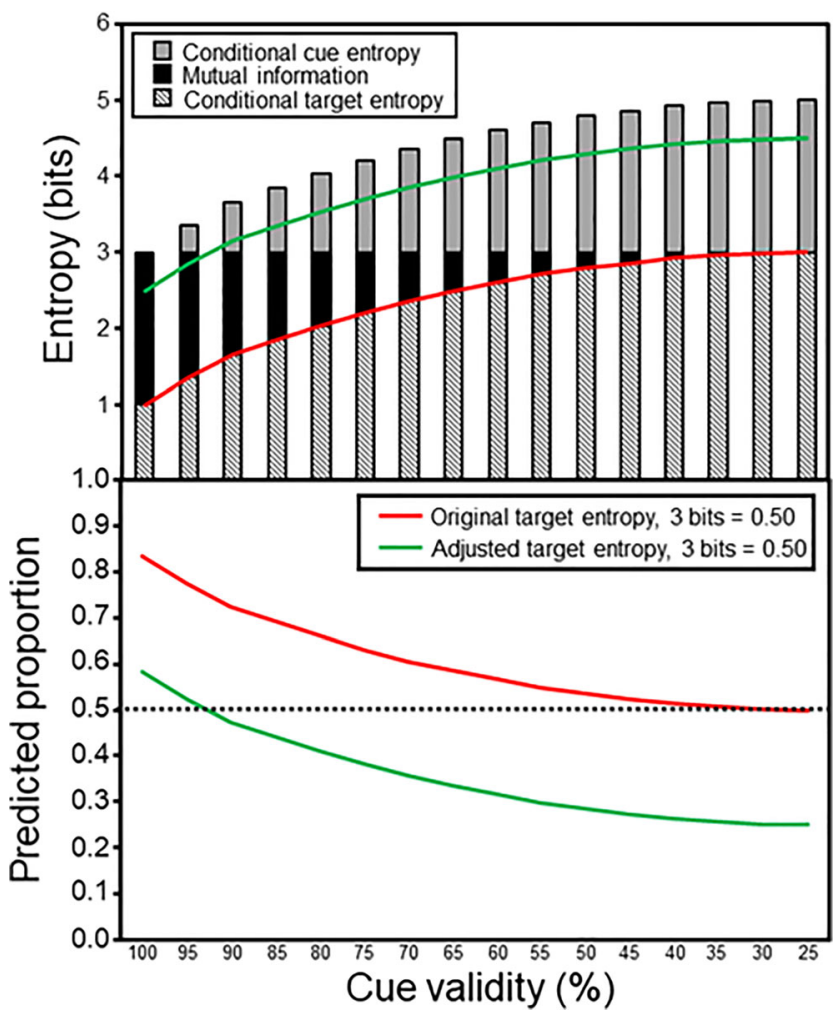

Fig. 9 Original and adjusted conditional target entropy curves (top panel), and original and adjusted predicted proportions (bottom panel), shown as a function of cue validity. The predicted proportions were based on Eqs. 6 and 7 (see main text)

entropy curve depicted in the upper panel of Fig. 9. This was accomplished by comparing two joint-distribution probability-cue contexts. In Experiment 4, participants could choose to search within the $25 \%$ valid cue context in which the target appeared at the cued location only by chance; or, they could choose to search within the $100 \%$ valid cue context in which the target always appeared at the cued location. This contrast was chosen based on examination of the newly adjusted predicted portions that resulted when the following equation was used to solve for the unknown proportion $x$ :

$$
\begin{gathered}
\frac{4.5 \text { bits }}{\text { Adjusted target entropy }}=\frac{0.50}{\text { Proportion } x} \\
\text { Proportion } x=\frac{0.50 \text { (Adjusted target entropy) }}{4.5 \text { bits }} .
\end{gathered}
$$

Notice that Eq. 9 differed from the Eq. 6 in that the 25\% valid cue context was now estimated to have 4.5 bits of target entropy as opposed to three bits of target entropy and the adjusted target entropy was now input as opposed to the original target entropy (compare the red and green lines in the upper panel of Fig. 9). Consequently, Eq. 9 was designed to return a predicted proportion of 0.50 for $x$ when the adjusted entropy of the target in the second search option was also equal to 4.5 bits. Finally, in accordance with Eq. 7 , the adjusted predicted proportion was obtained by taking the complement of the obtained value of $x$. The adjusted predicted proportions (using Eq. 9 with 4.5 bits $=0.50$ ) is represented by the green line in Fig. 10 and the original predicted proportions (using Eq. 6 with 3 bits $=0.50$ ) is represented by the red line. Clearly, the largest predicted difference between the adjusted and original conditional target entropy curves occurred when cue validity was 100\% - the chosen contrast in Experiment 4 .

According to the adjusted predicted proportions depicted in Fig. 10, participants should prefer the $100 \%$ valid cue context over the $25 \%$ valid cue context, with the observed proportion averaging approximately 0.72 . Furthermore, the $95 \% \mathrm{CI}$ drawn around the average observed proportion of " $100 \%$ valid cue" responses should include the adjusted predicted proportion of 0.72 , but not the original predicted proportion of 0.83 .

\section{Method}

Participants Forty individuals were recruited to participate through AMT in exchange for US\$5.00.

Stimuli and apparatus The stimuli and apparatus used in Experiment 4 were identical to those used in Experiment 2.

Procedure The procedure used in Experiment 4 was identical to the procedure used in Experiment 2, with the sole exception being that the $25 \%$ valid cue context served as one search option and the $100 \%$ valid cue context served as the other search option. Typical display sequences are depicted in the bottom-left panel of Fig. 3.

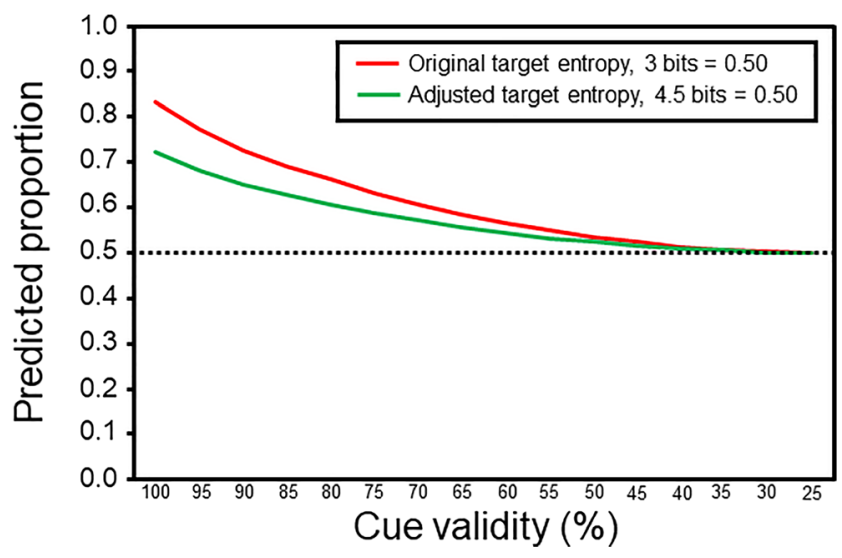

Fig. 10 Original and adjusted predicted proportions shown as a function of cue validity. The original predicted proportions are the same as those shown in Fig. 2 and were based on Eqs. 6 and 7; the adjusted predicted proportions were based on Eqs. 7 and 9 (see main text) 


\section{Results and discussion}

Task selection The proportion of " $100 \%$ valid cue" responses obtained in Experiment 4 is depicted in Fig. 11 as a function of trial block (blocks 1-4). An initial one-way, repeatedmeasures ANOVA was run to determine the extent to which the proportion of " $100 \%$ valid cue" responses varied across the four trial blocks. However, the main effect of trial block did not attain significance, $F(3,117)=1.11, p=.35, \eta^{2}=.03$. Consequently, we focused on the overall average proportion of " $100 \%$ valid cue" responses in the remainder of this experiment.

As expected, the results suggested a preference for the $100 \%$ valid cue context over the $25 \%$ valid cue context in that the proportion of " $100 \%$ valid cue" responses was significantly above chance $(M=0.74, S E=.04), t(39)=6.64, p<.001, d$ $=1.05$. More importantly, a 95\% CI computed around this average observed proportion (95\% CI: $0.66,0.81)$ included the adjusted predicted proportion of 0.72 (see the green line in Fig. 10), but not the original predicted portion of 0.83 (see the red line in Fig. 10). Thus, the results of Experiment 4 provided confirmatory evidence for the validity of the adjusted predicted proportions that were translated from the adjusted target entropy curves (Fig. 9).

Moreover, it is also worth pointing out that the observed proportion of " $100 \%$ valid cue" responses obtained in the present experiment was significantly greater than the observed proportion of " $100 \%$ valid cue" responses obtained in the $100 \%$ valid cue context of Experiment $2(M=0.61, S E=$ $.03), t(78)=2.44, p=.02, d=0.55$. The only difference between these two $100 \%$ valid cue contexts was that the $25 \%$ valid (joint-distribution) cue context was the alternative search context in Experiment 4 whereas the 25\% valid (singledistribution) location cue context was the alternative search context in Experiment 2. This modulation by task context is important because it further corroborates our interpretation that the lower-than-expected proportion of " $100 \%$ valid cue" responses obtained in the $100 \%$ valid cue context of Experiment 2 did not reflect a response bias against choosing

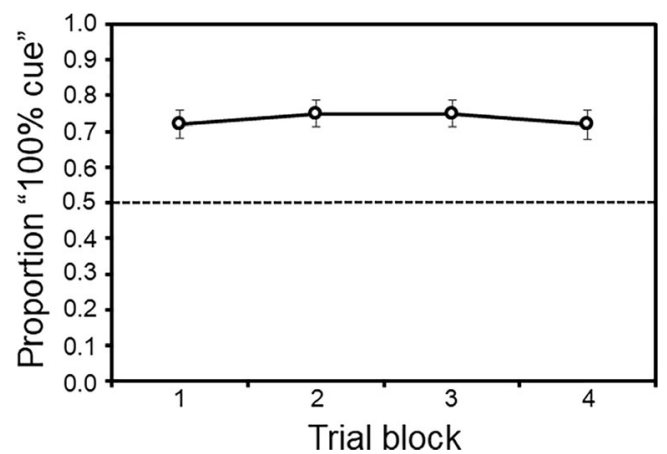

Fig. 11 The proportion of " $100 \%$ valid cue" responses obtained in Experiment 4 as a function of trial block. Error bars represent standard errors of the mean the task that appeared on the left side of the task selection screen. Such a bias would have predicted similar lower-thanexpected proportions of " $100 \%$ valid cue" responses across Experiments 2 and 4 had it been operating given that the same task was consistently mapped to the left side of the task selection screen across the two experiments, but this did not occur (see Table 3 for a summary).

Task performance Two participants were excluded from this analysis because they had exceptionally slow performance in at least one of the two cue contexts. For instance, one participant had mean correct RTs that were nearly six standard deviations slower than the group mean in the $100 \%$ valid cue context, whereas the other participant had mean correct RTs that were over four standard deviations slower than the group mean in the $25 \%$ valid cue context.

As expected, overall mean correct RTs were significantly faster in the $100 \%$ valid cue context $(M=725 \mathrm{~ms}, S E=44.15)$ than in the $25 \%$ valid cue context $(M=883 \mathrm{~ms}, S E=34.93)$, $t(37)=4.64, p<.001, d=.64$. However, although percent error rates were numerically smaller in the $100 \%$ valid cue context $(M=2.94 \%, S E=0.39)$ than in the $25 \%$ valid cue context $(M=5.35 \%, S E=1.38)$, this difference did not attain significance, $t(37)=1.75, p=.09, d=.39$. These taskperformance results are discussed further in the General discussion.

\section{General discussion}

The present study used information theory to quantify the extent to which single-distribution and joint-distribution probability cues reduced the uncertain identity and location of a visual search target. Although objective measures of entropy showed that single-distribution and joint-distribution probability cues reduced the uncertain identity and location of the target by equivalent amounts, the method of estimation was found to be more complex in the context of joint-distribution probability cues relative to single-distribution probability cues (see Tables 1 and 2; see also Fig. 1). In particular, the conditional form of target entropy associated with joint-distribution probability cues was considered to be more complex because this quantity needed to be partitioned from mutual information and conditional cue entropy in the joint probability distribution, whereas the simpler form of target entropy associated with single-distribution probability cues was considered to be less complex because this quantity could be estimated directly from the target identity and location probability distribution.

The present study inferred the extent to which this difference in complexity led to differences in the representation of uncertainty by examining the extent to which individuals explicitly preferred one form of expected uncertainty over the 
other within the context of a novel spatial cue variant of the demand selection paradigm. In particular, participants were offered the choice between two visual search tasks which differed only in the type and/or validity of the probability cue. Furthermore, participants selected their preferred task context explicitly at the beginning of each trial. The use of a demand selection paradigm in the present study was important because latency has served as the de facto measure of cue use in previous spatial cueing studies, especially the magnitude of the latency difference between valid and invalid trials (Chica et al., 2014); however, latency measures are inherently relative in nature and cannot provide an estimate of absolute rates of spatial cue use (e.g., Blair, Capozzi, \& Ristic, 2017; Davis \& Gibson, 2012; Pauszek \& Gibson, 2016). In addition, latency does not provide useable information regarding observers' preferences, nor their explicit intention to use a cue or not, though latency measures may inform explanations involving the minimization of effort and/or the maximization of reward rate (see below for further discussion).

Within the context of this demand selection paradigm, the main results suggested representation of greater target entropy for joint- than for single-distribution cues based on a comparison between predicted and observed probability cue choices across four experiments. In particular, the target entropy curves shown in Fig. 1 were translated to the predicted proportions in Fig. 2 using Eqs. 6 and 7 in which the 25\% valid single-distribution probability-cue context served as the baseline. Experiment 1 showed good correspondence between the observed and predicted proportions (i.e., the predicted proportion fell within the 95\% CI computed around the observed proportion) when participants compared two singledistribution probability-cue contexts (i.e., when they compared the $70 \%$ valid location context with the $25 \%$ valid location baseline).

However, Experiments 2 and 3 showed poor correspondence between the observed and predicted proportions when participants compared one of three jointdistribution probability-cue contexts (i.e., either the $100 \%, 70 \%$, or $25 \%$ valid cue context) with the $25 \%$ valid location baseline. In each of these joint-distribution probability-cue contexts, the observed proportion of "joint-distribution cue" responses was approximately 0.25 less than predicted, which in turn implied that joint-distribution probability cues conveyed approximately 1.5 more bits of uncertainty than the corresponding single-distribution probability cues (compare the green and red lines in the top panel of Fig. 9). In other words, the $100 \%$ valid jointdistribution probability cue was estimated to convey 2.5 bits (as opposed to $1.0 \mathrm{bit}$ ) of information, the $70 \%$ valid joint-distribution probability cue was estimated to convey 3.86 bits (as opposed to 2.36 bits) of information, and the $25 \%$ valid joint-distribution probability cue was estimated to convey 4.5 bits (as opposed to 3.0 bits) of information.
Of course, the observed versus predicted difference that was obtained in Experiments 2 and 3 did not demand an interpretation that was rooted in the representation of uncertainty. For instance, the obtained difference could have reflected a preference for random search over deliberate, top-down search (see, e.g., Wolfe, Alvarez, \& Horowitz, 2000), though the results showing that participants preferred searching within (1) the $70 \%$ valid location context over the $25 \%$ valid location context in Experiment 1; (2) the 100\% valid cue context over the 25\% valid location context in Experiments 2 and 3; and (3) the $100 \%$ valid cue context over the $25 \%$ valid cue context in Experiment 4 were all inconsistent with the notion that task selection reflected a general preference for random search.

Alternatively, the observed versus predicted difference could have reflected a preference for the "least cost" option over a "greater cost" option (see, e.g., Pauszek \& Gibson, 2016, 2018, 2019). For instance, the information conveyed by joint-distribution probability cues required processing the changing direction of a physical symbol - an arrow - on every trial, whereas the information conveyed by single-distribution probability cues did not. In this way, task choice may have been modulated by the greater cost of processing the cue in the joint-distribution context relative to the single-distribution context. However, it is reasonable to expect that the cost of searching with a joint-distribution probability cue should have been modulated by the validity of that cue as well as by the difficulty of searching without that cue, but Experiments 2 and 3 provided no evidence of this modulation. Instead, the observed versus predicted difference remained relatively constant across cue validity and task difficulty.

In addition, Pauszek (2019, unpublished dissertation) obtained results that were also inconsistent with the notion that task choices were modulated by cue processing costs (Pauszek, 2019). This experiment was identical to Experiment 2 of the present study with the sole exception being that it used $70 \%$ valid spatial word cues instead of $70 \%$ valid arrow cues (40 undergraduate participants served in this experiment; the alternative search task was the $25 \%$ valid location context; and, three distractors were present in the target display). The spatial word cues conveyed the same four directions as the arrow cues; however, the spatial relations expressed by spatial word cues such as "above," "below," "left," and "right" are thought to be more complex and difficult-to-process than corresponding arrow cues (e.g., Carlson, 2003; Coventry \& Garrod, 2004; CarlsonRadvansky \& Jiang, 1998; Carlson \& Van Deman, 2004, 2008; Garnham, 1989; Gibson \& Kingstone, 2006; Gibson, Scheutz, \& Davis, 2009; Kemmerer, 2006; Logan, 1995; Pauszek \& Gibson, 2018, 2019).

Thus, there was good reason to expect that the proportion of "joint-distribution probability cue" responses would be significantly less when spatial word cues were shown, had task 
selection been influenced by cue processing difficulty. However, the proportion of "joint-distribution probability cue" responses was observed to be $M=0.29$ ( $S E=0.04$; 95\% CI: 0.22, 0.36 ) in Pauszek's experiment, and this proportion did not differ significantly from the proportion observed under similar conditions in Experiment 2 when $70 \%$ valid arrow cues were shown $(M=0.30 ; S E=0.03), t(78)=$ $0.32, p=.75, d=0.07$. Hence, the present pattern of preferences has been shown to generalize beyond arrow cues, and this replication is consistent with the notion that the task selection was based primarily on differences in the representation of target entropy, not differences in cue processing costs.

As mentioned in the Introduction, another manifestation of the effort minimization and/or reward maximization accounts is that participants may prefer task contexts that can be completed faster and/or more accurately, regardless of the uncertainty conveyed by the probability cues. Accordingly, mean correct RTs and percent error rates were also assessed in each of the four experiments to examine the adequacy of these accounts. Indeed, the present findings suggested that participants tended to prefer search contexts, such as the $70 \%$ valid location context of Experiment 1 and the 100\% valid cue contexts of Experiments 2, 3, and 4, that were also performed overall faster (and, in some cases, more accurately) than the alternative search context. However, this evidence was offset by several findings which suggested that participants could also develop preferences for search contexts, such as the $25 \%$ valid location contexts of Experiments 2 and 3, even when those contexts did not result in overall faster or more accurate performance (relative to the $70 \%$ valid and $25 \%$ valid cue contexts). Thus, the experience of an overall task performance advantage does not appear to be a necessary factor in the development of a search task preference. Furthermore, this interpretation is consistent with other recent studies that have found surprisingly high rates of non-optimal visual search strategy choices (see, e.g., Irons \& Leber, 2016), leading to the conclusion that performance maximization and/or effort minimization alone are unable to fully account for individuals' strategic visual search preferences.

The strongest evidence that the observed versus predicted difference obtained in Experiments 2 and 3 was rooted in the misrepresentation of entropy came from Experiment 4, which compared two joint-distribution probability-cue contexts: the $100 \%$ valid cue context versus the $25 \%$ valid cue context. Under these conditions, cue type was controlled, and there should have been good correspondence between the original predicted proportions (see the red line in Figs. 2 and 10) and the observed proportions, had participants represented the uncertainty of joint-distribution probability cues to be equivalent to the uncertainty of single-distribution probability cues. However, the results showed that there was poor correspondence between the observed and predicted proportions when the original target entropy estimates were used to derive the predicted proportions. In contrast, there was good correspondence between the observed and predicted proportions only when adjusted target entropy estimates were used to derive the predicted proportions (see the green line in Fig. 10). These findings therefore provided stronger evidence for the interpretation that participants represented the uncertainty of jointdistribution probability cues to be greater than the uncertainty of single-distribution probability cues.

Moreover, the observed proportion of " $100 \%$ valid cue" responses obtained in Experiment 4 was significantly greater than the observed proportion obtained in Experiment 2, even though the 158-ms overall task performance benefit associated with this context in Experiment 4 was very similar to the 175 -ms overall task performance benefit associated with this context in Experiment 2. As mentioned above, the primary difference between these two $100 \%$ valid cue contexts was that the $25 \%$ valid (joint-distribution) cue context was the alternative search context in Experiment 4 whereas the 25\% valid (single-distribution) location cue context was the alternative search context in Experiment 2. Consistent with a representational basis, this difference occurred because the target entropy in the $100 \%$ valid cue context was estimated to be 2.5 bits, whereas the target entropy in the $25 \%$ valid cue context was estimated to be 4.5 bits and the target entropy in the $25 \%$ valid location context was estimated to be 3.0 bits. Thus, the relative difference in target entropy was greater between the $100 \%$ valid cue context and the $25 \%$ valid cue context of Experiment 4 (see the green line in Fig. 10, which predicted a proportion of 0.72 ) than it was between the $100 \%$ valid cue context and the $25 \%$ valid location context of Experiment 2 (see the green line in the bottom panel of Fig. 9, which predicted a proportion of 0.58 ).

The findings obtained in the present study suggested that the representation of basic information-theoretic quantities such as entropy can depend on the extent to which those quantities were based on single versus joint probability distributions. This finding is important because informationtheoretic quantities such as mutual information and conditional entropy serve as the building blocks of higher-level, decision-theoretic processes involving Bayesian inference or active inference (see, e.g., Bogacz, 2017; Clark, 2016; Friston, 2009, 2013; Gershman, 2019; Hutchinson \& Barrett, 2019, for a range of perspectives). For instance, both Bayesian inference and active inference approaches to perception, cognition, and action predict that individuals tend to enact behavioral policies that maximize mutual information and/or minimize entropy. By showing that the representation of basic information-theoretic quantities can depend on the complexity of the underlying probability distributions, the present findings serve to show that the representation of mutual information and/or entropy need not always correspond to the mathematically defined entities. Given the growing importance of Bayesian inference and active inference approaches to 
perception, cognition, and action, the present findings therefore suggest that more research needs to be focused on how individuals process the information-theoretic quantities that underlie these approaches.

That said, more evidence will be needed to increase confidence in the conclusion that the present findings were due to differences in how entropy was estimated from single versus joint probability distributions. One issue concerns the source of the misrepresentation of conditional target entropy in the context of joint-distribution probability cues. The present findings suggested that the magnitude of misrepresentation associated with the joint-distribution probability cues remained relatively constant across the entire range of cue validity, which was interpreted to reflect inabilities to both estimate mutual information (at higher cue validities) and to partition conditional cue entropy from conditional target entropy (at lower cue validities). However, future studies will need to examine why individuals' observed performance deviated from predictions based on the three information-theoretic quantities associated with joint-distribution probability cues. The regularities obtained here should also serve as "targets" for a principled process model that can account for these regularities. Our conclusions here should be read in terms of the regularities described in our information theory account, not in terms of any specific hypotheses about the processes of internal computation.

A second issue that should be examined more closely concerns the extent to which the present conclusions generalize to other joint-distribution probability cues. As discussed above, we have obtained similar findings with both arrow cues and spatial word cues. However, a stronger challenge to the present conclusion involves the distinction between so-called "endogenous" and "exogenous" spatial cues. Endogenous cues are typically symbols that refer to spatial locations indirectly - such as when an arrow appears at one location (fixation) and points to another location (in the visual periphery), whereas exogenous cues are typically stimuli that refer to spatial locations more directly - such as when a square outline suddenly appears at one of the potential target locations in the visual periphery (Yantis, 1996). Although there has been intense debate over the past 30 years concerning the extent to which exogenous cues might control attention in a more stimulusdriven or bottom-up fashion than endogenous cues (see, e.g., Corbetta \& Shulman, 2002; Folk, Remington, \& Johnston, 1992; Gibson \& Kelsey, 1998; Jonides, 1981; Mayer, Dorflinger, Rao, \& Seidenberg, 2004; Müller \& Findlay, 1988; Müller \& Rabbitt, 1989; Posner \& Petersen, 1990; Posner \& Snyder, 1975; Yantis \& Jonides, 1990; see also, Wright \& Ward, 2008, for a review), objective measures of the magnitude and complexity of the target entropy conveyed by both of these cues should be equivalent. In other words, the target entropy conveyed by both endogenous and exogenous cues would be based on the same conditional form of target entropy, and presumably estimated only after it had been partitioned from mutual information and conditional cue entropy in the joint probability distribution (see Table 2 and Fig. 1). As such, endogenous and exogenous cues would both be considered to be joint-distribution probability cues.

Thus, if task selection in the present demand selection paradigm is influenced primarily by represented target entropy, and if represented target entropy is influenced primarily by the complexity of the underlying estimation, then the represented entropy of exogenous cues should be similar to the represented entropy of endogenous cues. Furthermore, the represented entropy of both of these cues should be greater than the represented entropy of single-distribution probability cues. Such findings would be important because they would support a new taxonomy of spatial cues that is founded on information theory, and would presumably extend beyond the binary distinction between single versus joint probability distributions (cf. Hommel et al., 2019). However, this issue is beyond the scope of the present study and must await future research to be resolved.

The present study returned to an approach that was inspired by Charles Eriksen, using information theory to quantify the extent to which single-distribution and joint-distribution probability cues reduced the uncertain identity and location of a visual search target. Although objective measures of entropy showed that single-distribution and joint-distribution probability cues reduced the uncertain identity and location of the target by equivalent amounts, the method of estimation was found to be more complex in the context of joint-distribution probability cues relative to single-distribution probability cues. The present study attempted to infer the extent to which this difference in complexity led to differences in the representation of uncertainty by examining the extent to which individuals explicitly preferred one form of expected uncertainty over the other within the context of a demand selection paradigm. Within the context of this paradigm, the present study concluded that the representation of target entropy was greater for joint- than for single-distribution cues.

Open Practices Statement The data and materials for all experiments are available from the first author upon request and none of the experiments were preregistered.

\section{References}

Beck, J.M., Ma, W.J., Pitkow, X., Latham, P.E., \& Pouget, A. (2012). Not noisy, just wrong: The role of suboptimal inference in behavioral variability. Neuron, 74, 30-39.

Blair, C.D., Capozzi, F., \& Ristic, J. (2017). Where is your attention? Assessing individual instances of covert attentional orienting in response to gaze and arrow cues. Vision, 1(3), 19. 
Bland, A.R., \& Schaefer, A. (2012). Unexpected uncertainty, volatility and decision-making. Frontiers in Neuroscience, 6, 00085.

Bogacz, R. (2017). A tutorial on the free-energy framework for modelling perception and learning. Journal of Mathematical Psychology, 76, 198-211.

Bogacz, R., Brown, E., Moehlis, J., Holmes, P., \& Cohen, J.D. (2006). The physics of optimal decision making: A formal analysis of models of performance in two-alternative forced-choice tasks. Psychological Review, 113, 700-765.

Carlson, L.A. (2003). Using spatial language. The Psychology of Learning and Motivation, 43, 127-161.

Carlson, L.A., \& Van Deman, S.R. (2004). The space in spatial language. Journal of Memory and Language, 51, 418-436.

Carlson, L.A., \& Van Deman, S.R. (2008). Inhibition with a reference frame during the interpretation of spatial language. Cognition, 106, 384-407.

Carlson-Radvansky, L.A., \& Jiang, Y. (1998). Inhibition accompanies reference-frame selection. Psychological Science, 9, 386-391.

Chica, A.B., Martín-Arévalo, E., Botta, F., \& Lupiáñez, J. (2014). The spatial orienting paradigm: How to design and interpret spatial attention experiments. Neuroscience and Biobehavioral Reviews, 40, 35-51.

Cisek, P. (2019). Resynthesizing behavior through phylogenetic refinement. Attention, Perception, \& Psychophysics, 81, 2265-2287.

Clark, A. (2016). Surfing uncertainty: Prediction, action, and the embodied mind. New York: Oxford University Press.

Colgate, R.L., Hoffman, J.E., \& Eriksen, C.W. (1973). Selective encoding from multielement visual displays. Perception \& Psychophysics, 14, 217-224.

Corbetta, M., \& Shulman, G.L. (2002). Control of goal-directed and stimulus-driven attention in the brain. Nature Reviews Neuroscience, 3, 215-229.

Coventry, K.R., \& Garrod, S.C. (2004). Saying, seeing, and acting: The psychological semantics of spatial prepositions. New York: Psychology Press.

Cover, T.M., \& Thomas, J.A. (2006). Elements of information theory. New York: Wiley.

Crump, M.J.C., McDonnell, J.V., \& Gureckis, T.M. (2013). Evaluating Amazon's Mechanical Turk as a tool for experimental behavioral research. PLOS ONE, 8(3), 1-18.

Davis, G.J., \& Gibson, B.S. (2012). Going rogue in the spatial cuing paradigm: High spatial validity is insufficient to elicit voluntary shifts of attention. Journal of Experimental Psychology: Human Perception and Performance, 38, 1192-1201.

Eriksen, C.W. (1952). Location of objects in a visual display as a function of the number of dimensions on which the objects differ. Journal of Experimental Psychology, 44, 55-61.

Eriksen, C.W. (1953). Object location in a complex visual field. Journal of Experimental Psychology, 45, 126-132.

Eriksen, C.W. (1955). Partitioning and saturation of visual displays and efficiency of visual search. Journal of Applied Psychology, 39, 7377.

Eriksen, C.W., \& Hake, H.W. (1955a). Absolute judgments as a function of stimulus range and number of stimulus and response categories. Journal of Experimental Psychology, 49, 323-332.

Eriksen, C.W., \& Hake, H.W. (1955b). Multidimensional stimulus differences and accuracy of discrimination. Journal of Experimental Psychology, 50, 153-160.

Eriksen, C.W., \& Hoffman, J.E. (1972). Temporal and spatial characteristics of selective encoding from visual displays. Perception \& Psychophysics, 12, 201-204.

Folk, C.L., Remington, R.W., \& Johnston, J.C. (1992). Involuntary covert orienting is contingent on attentional control settings. Journal of Experimental Psychology: Human Perception and Performance, $18,1030-1044$
Forster, K.I., \& Forster, J.C. (2003). DMDX: A Windows display program with millisecond accuracy. Behavior Research Methods: Instruments and Computers, 35, 116-124.

Friston, K. (2009). The free-energy principle: A rough guide to the brain? Trends in Cognitive Sciences, 13(7), 293-301.

Friston, K. (2013). Life as we know it. Journal of The Royal Society Interface, 10(86), 1-12.

Garnham, A. (1989). A unified theory of the meaning of some spatial relational terms. Cognition, 31, 45-60.

Geng, J.J., \& Behrmann, M. (2002). Probability cuing of target location facilitates visual search implicitly in normal participants and patients with hemispatial neglect. Psychological Science, 13(6), 520-525.

Geng, J.J., \& Behrmann, M. (2005). Spatial probability as an attentional cue in visual search. Perception \& Psychophysics, 67(7), 12521268.

Gershman, S.J. (2019). What does the free energy principle tell us about the brain? arXiv: 1901.0794.

Gibson, B.S., \& Davis, G.J. (2011). Grounding spatial language in the motor system: Reciprocal interactions between conceptual control and spatial orienting. Visual Cognition, 19, 79-116.

Gibson, B.S., \& Kelsey, E.M. (1998). Stimulus-driven attentional capture is contingent on attentional set for displaywide visual features. Journal of Experimental Psychology: Human Perception and Performance, 24(3), 699-706.

Gibson, B.S., \& Kingstone, A.F. (2005). Visual attention and the semantics of space: Beyond central and peripheral cues. Paper presented at the $46^{\text {th }}$ annual meeting of the Psychonomic Society, Toronto.

Gibson, B.S., \& Kingstone, A.F. (2006). Visual attention and the semantics of space: Beyond central and peripheral cues. Psychological Science, 17, 622-627.

Gibson, B.S., Scheutz, M., \& Davis, G.J. (2009). Symbolic control of visual attention: Semantic constraints on the spatial distribution of attention. Attention, Perception, \& Psychophysics, 71, 363-374.

Hake, H.W., \& Eriksen, C.W. (1955). Effect of number of permissible response categories on learning of a constant number of visual stimuli. Journal of Experimental Psychology, 50(3), 161-167.

Hommel, B., Chapman, C.S., Cisek, P., Nevedli, H.F., Song, J.H., \& Welsh, T.N. (2019). No one knows what attention is. Attention, Perception, \& Psychophysics, 81(7), 2288-2303.

Hutchinson, J.B., \& Barrett, L.F. (2019). The power of predictions: An emerging paradigm for psychological research. Current Directions in Psychological Science, 28(3), 280-291.

Irons, J.L., \& Leber, A.B. (2016). Choosing attentional control settings in a dynamically changing environment. Attention, Perception, \& Psychophysics, 78(7), 2031-2048.

Jiang, Y.V. (2018). Habitual versus goal-driven attention. Cortex, 102, 107-120.

Jiang, Y.V., Swallow, K.M., \& Rosenbaum, G.M. (2013a). Guidance of spatial attention by incidental learning and endogenous cuing. Journal of Experimental Psychology: Human Perception and Performance, 39(1), 285-297.

Jiang, Y.V., Swallow, K.M., Rosenbaum, G.M., \& Herzig, C. (2013b). Rapid acquisition but slow extinction of an attentional bias in space. Journal of Experimental Psychology: Human Perception and Performance, 39(1), 87-99.

Jonides, J. (1981). Voluntary versus automatic control over the mind's eye movement. In J.B. Long \& A.D. Baddeley (Eds.), Attention and performance $I X$ (187-203). Hillsdale: Erlbaum.

Kemmerer, D. (2006). The semantics of space: Integrating linguistic typology and cognitive neuroscience. Neuropsychologia, 44, 16071621.

Kool, W., McGuire, J.T., Rosen, Z.B., \& Botvinick, M.M. (2010). Decision making and the avoidance of cognitive demand. Journal of Experimental Psychology: General, 139(4), 665-682.

Logan, G.D. (1995). Linguistic and conceptual control of visual spatial attention. Cognitive Psychology, 28, 103-174. 
Luce, R. D. (2003). Whatever happened to information theory in psychology? Review of General Psychology, 7, 183-188.

Ma, W.J. (2012). Organizing probabilistic models of perception. Trends in Cognitive Science, 16, 511-518.

Mayer, A.R., Dorflinger, J.M., Rao, S.M., \& Seidenberg, M. (2004). Neural networks underlying endogenous and exogenous visualspatial orienting. Neurolmage, 23(2), 534-541.

Müller, H.J., \& Findlay, J.M. (1988). The effect of visual attention on peripheral discrimination thresholds in single and multiple element displays. Acta Psychologica, 69, 129-155.

Müller, H.J., \& Rabbitt, P.M.A. (1989). Reflexive and voluntary orienting of visual attention: time course of activation and resistance to interruption. Journal of Experimental Psychology: Human Perception and Performance, 15(2), 315-330.

Pauszek, J.R. (2019). A predictive coding account of attention control (Doctoral dissertation). Retrieved from University of Notre Dame's CurateND (Record 005074341).

Pauszek, J.R., \& Gibson, B.S. (2016). High spatial validity is not sufficient to elicit voluntary shifts of attention. Attention, Perception, \& Psychophysics, 78(7), 2110-2123.

Pauszek, J.R., \& Gibson, B.S. (2018). The least costs hypothesis: A rational analysis approach to the voluntary symbolic control of attention. Journal of Experimental Psychology: Human Perception and Performance, 44(8), 1199-1215.

Pauszek, J.R., \& Gibson, B.S. (2019). Dealing with distractors in the spatial cueing paradigm can reflect the strategic influence of cognitive effort minimization rather than a limit to selective attention. Visual Cognition, 27(3-4), 367-383.

Pauszek, J.R., Sztybel, P., \& Gibson, B.S. (2017). Evaluating Amazon's Mechanical Turk for psychological research on the symbolic control of attention. Behavior Research Methods, 49(6), 1969-1983.

Posner, M.I. (1980). Orienting of attention. Quarterly Journal of Experimental Psychology, 32, 3-25.

Posner, M.I., \& Petersen, S.E. (1990). The attention system of the human brain. Annual Review of Neuroscience, 13, 25-42.
Posner, M.I., \& Snyder, C.R. (1975). Attention and cognitive control. In R.L. Solso (Ed.), Information processing and cognition: The Loyola symposium (55-85). Hillsdale: Erlbaum.

Pouget, A., Beck, J.M., Ma, W.J., Latham, P.E. (2013). Probabilistic brains: Knowns and unknowns. Nature Neuroscience, 16, 11701178 .

Shaw, M.L., \& Shaw, P. (1977). Optimal allocation of cognitive resources to spatial locations. Journal of Experimental Psychology: Human Perception and Performance, 3, 201-211.

Stone, J.V. (2015). Information theory: A tutorial introduction. England: Sebtel Press.

Witzel, J., Cornelius, S., Witzel, N., Forster, K.I., \& Forster, J.C. (2013). Testing the viability of webDMDX for masked priming experiments. The Mental Lexicon, 8, 421-449.

Wolfe, J.M., Alvarez, G.A., \& Horowitz, T.S. (2000). Attention is fast but volition is slow. Nature, 406(6797), 691.

Wright, R.D., \& Ward, L.M. (2008). Orienting of attention. New York: Oxford University Press.

Yantis, S. (1996). Attentional capture in vision. In A.F. Kramer, M.G.H. Coles, \& G.D. Logan (Eds.), Converging operations in the study of visual selective attention (45-76). Washington: American Psychological Association.

Yantis, S., \& Jonides, J. (1990). Abrupt visual onsets and selective attention: Voluntary versus automatic allocation. Journal of Experimental Psychology: Human Perception and Performance, 16, 121-134.

$\mathrm{Yu}$, A.J., \& Dayan, P. (2005). Uncertainty, neuromodulation, and attention. Neuron, 46(4), 681-692.

Zhang, Z., \& Yuan, K.-H. (2015). WebPower: Statistical power analysis online. Retrieved from https://webpower.psychstat.org

Publisher's note Springer Nature remains neutral with regard to jurisdictional claims in published maps and institutional affiliations. 